\title{
Myelin repair stimulated by CNS-selective thyroid hormone action
}

\author{
Meredith D. Hartley, ${ }^{1,2}$ Tania Banerji, ${ }^{1}$ Ian J. Tagge, ${ }^{3}$ Lisa L. Kirkemo, ${ }^{1,2}$ Priya Chaudhary, ${ }^{2,4}$ \\ Evan Calkins, ${ }^{2,4}$ Danielle Galipeau,, ${ }^{2,4}$ Mitra D. Shokat, ${ }^{1}$ Margaret J. DeBell, ${ }^{1}$ Shelby Van Leuven, ${ }^{1}$ \\ Hannah Miller, ${ }^{1}$ Gail Marracci,, ${ }^{2,4}$ Edvinas Pocius,, ${ }^{2,4}$ Tapasree Banerji, ${ }^{1}$ Skylar J. Ferrara, ${ }^{1}$ \\ J. Matthew Meinig, ${ }^{1}$ Ben Emery, ${ }^{4,5}$ Dennis Bourdette, ${ }^{2,4}$ and Thomas S. Scanlan ${ }^{1}$ \\ 'Department of Physiology \& Pharmacology and Program in Chemical Biology, Oregon Health \& Science University, \\ Portland, Oregon, USA. ${ }^{2}$ VA Portland Health Care System, Portland, Oregon, USA. ${ }^{3}$ Advanced Imaging Research Center, \\ ${ }^{4}$ Department of Neurology, and 5 Jungers Center for Neurosciences Research, Oregon Health \& Science University, \\ Portland, Oregon, USA.
}

Oligodendrocyte processes wrap axons to form neuroprotective myelin sheaths, and damage to myelin in disorders, such as multiple sclerosis (MS), leads to neurodegeneration and disability. There are currently no approved treatments for MS that stimulate myelin repair. During development, thyroid hormone $(\mathrm{TH})$ promotes myelination through enhancing oligodendrocyte differentiation; however, TH itself is unsuitable as a remyelination therapy due to adverse systemic effects. This problem is overcome with selective TH agonists, sobetirome and a CNS-selective prodrug of sobetirome called Sob-AM2. We show here that TH and sobetirome stimulated remyelination in standard gliotoxin models of demyelination. We then utilized a genetic mouse model of demyelination and remyelination, in which we employed motor function tests, histology, and MRI to demonstrate that chronic treatment with sobetirome or Sob-AM2 leads to significant improvement in both clinical signs and remyelination. In contrast, chronic treatment with TH in this model inhibited the endogenous myelin repair and exacerbated disease. These results support the clinical investigation of selective CNS-penetrating TH agonists, but not TH, for myelin repair.

Conflict of interest: MDH, Tania Banerij, PC, GM, Tapasree Banerij, SJF, JMM, DB, and TSS are inventors on licensed pending patents (Wo/2014/178931, W0/2016/134292, and W0/2018/032012). TSS, DB, and $B E$ are founders of Llama Therapeutics Inc. MDH is a consultant to Llama Therapeutics Inc.

Copyright: (c) 2019 American Society for Clinical Investigation

Submitted: November 20, 2018 Accepted: March 12, 2019 Published: April 18, 2019

Reference information: /CI Insight. 2019;4(8):e126329. https://doi. org/10.1172/jici.nsight.126329

\section{Introduction}

Demyelination of axons in the CNS is a primary feature of multiple sclerosis (MS) and a large family of genetic leukodystrophies. Mature oligodendrocytes extend and wrap processes around axons forming protective myelin sheaths. The oligodendrocyte pool arises from differentiation of oligodendrocyte progenitor cells (OPCs), which are present both during development and in the adult CNS. The final critical differentiation step becomes impaired in MS evidenced by the presence of OPCs in demyelinating lesions in MS that do not develop into myelin-producing mature oligodendrocytes $(1,2)$. Accordingly, there is a clinical need for therapeutic agents that can stimulate endogenous OPC differentiation, which may lead to remyelination and repair of the demyelinating lesions in MS.

One approach to this goal based on promoting OPC differentiation involves exploiting thyroid hormone (TH) action in the CNS, which is critical in developmental myelination (3-7). Indeed, the active form of $\mathrm{TH}$, 3,5,3'-triiodothyronine (T3), is routinely used as a positive control in OPC differentiation screening assays (8-10) and has been found to enhance myelin repair in several in vivo demyelination models (11-18). However, TH has not been pursued clinically, because chronic elevated systemic TH exposure, or hyperthyroidism, adversely affects heart, bone, and skeletal muscle, thus limiting its potential as a remyelination therapy (19).

$\mathrm{TH}$ agonists, or thyromimetics, are a class of compounds that mimic T3 binding at the $\mathrm{TH}$ receptor (TR). Sobetirome is a clinical-stage thyromimetic devoid of the adverse effects associated with hyperthyroidism and unique among thyromimetics for its ability to cross the blood-brain barrier and distribute to the CNS from a systemic dose $(20,21)$. Sobetirome has been shown to stimulate $\mathrm{TH}$ actions during brain development $(22,23)$ and alter brain lipid biomarkers in a model of X-linked adrenoleukodystrophy (24). The increased safety margin of sobetirome compared with TH arises from a combination of differential tissue distribution and specificity for TR $\beta$ over TR $\alpha$ (19). Thus, sobetirome appears to have the requisite properties and therapeutic index needed for stimulating myelin repair via a $\mathrm{TH}$ mechanism of action. 
In this study we tested the effect of different modes of $\mathrm{TH}$ action in different models of demyelination. Using an in vitro model of OPC differentiation and in vivo gliotoxin models of demyelination, we demonstrated that sobetirome mimics T3 to promote OPC differentiation and myelin repair in short-term disease settings. We then employed a chronic model of demyelination, in which deletion of myelin regulatory factor (Myrf) transcription factor resulted in pervasive CNS demyelination over a 10- to 12-week period followed by gradual but incomplete myelin repair. In this genetic model, thyromimetic action promoted brain and spinal cord myelin repair and correlated with significant improvement in neurological clinical signs that arise from CNS demyelination. Further, we found that chronic administration of T3 was not tolerated in this model and inhibited proliferation of OPCs, emphasizing the need for selective thyromimetic action. This study provides a model for assessing pro-remyelinating therapies that has advantages over existing models and supports clinical development of selective thyromimetics for treating MS.

\section{Results}

Sobetirome mimics T3 action in OPCS and in gliotoxin models of demyelination. TH is used to induce differentiation of cultured OPCs into mature oligodendrocytes $(25,26)$, and sobetirome has been shown to induce differentiation of mouse OPCs (23). We have replicated this finding with sobetirome using rat OPCs (Figure 1, A and B). TH action induced differentiation via transcriptional regulation of genes involved in myelination, including $M b p(27), K l f 9$ (28), and $H r(29)$. In rat OPCs treated for 1 day with T3 or sobetirome, all 3 genes were upregulated relative to vehicle controls (Figure 1, C-E). Withdrawal of PDGF induced differentiation of OPCs separately from $\mathrm{TH}$, but $\mathrm{TH}$ action on all 3 genes was observed both in the presence and absence of PDGF. TH-dependent gene regulation was also observed in mice, which showed upregulation of both Klf9 and $H r$ in the brain, after treatment for 7 days with T3 or sobetirome (1 mg/kg/d, i.p.) (Supplemental Figure 1A; supplemental material available online with this article; https://doi.org/10.1172/jci.insight.126329DS1). Upregulation was observed in the cerebellum, corpus callosum, hippocampus, and striatum (Supplemental Figure 1, B-E). Thus, systemically dosed sobetirome accessed and induced a biological response in brain tissue, including oligodendrocyte-rich white matter regions with high myelin content.

We next assessed whether sobetirome could stimulate myelin repair in vivo. To induce focal demyelination, we injected lysolecithin (2\%) into the corpus callosum (Figure 2A and Supplemental Figure 2, A and B) (30) and initiated once-daily injections with vehicle, T3 (1 mg/kg/d, i.p.), or sobetirome (1 or $5 \mathrm{mg} /$ $\mathrm{kg} / \mathrm{d}$, i.p.) 5 days later during the OPC recruitment phase of remyelination. The lesion volume was estimated by staining sequential sections through the lesion with the myelin stain BlackGold (Supplemental Figure 2C). In control mice, the lesion volume increased from day 5 through day 12 and then rapidly decreased by day 15 (Figure 2B). In contrast, the average lesion volume at day 12 was significantly lower in mice administered T3 or sobetirome (Figure 2, B and C), suggesting that TH action hastened lesion repair.

We also tested the effects of $\mathrm{T} 3$ and sobetirome in a model with more extensive demyelination in which mice were administered chow containing $0.2 \%$ cuprizone for 12 weeks. The mice were then returned to normal chow and received 3 weeks of once-daily injections with vehicle, T3 ( $1 \mathrm{mg} / \mathrm{kg} / \mathrm{d}$, i.p.), or sobetirome (1 mg/kg/d, i.p.). Three brain regions (cortex, hippocampus, and corpus callosum) were evaluated. Consistent with previous reports $(11,12,16,17,31)$, treatment with T3 resulted in elevated numbers of ASPA-positive oligodendrocytes and increased myelin basic protein (MBP) staining in the hippocampus and cortex relative to that in controls (Figure 2, D-F, and Supplemental Figure 3, A, B, G, and H) but no changes in OPC levels (Supplemental Figure 3, C, D, F, I, J, and L). Treatment with sobetirome increased oligodendrocyte numbers and MBP staining to a lesser extent than treatment with T3 (Figure 2, E and F). Analysis of the caudal corpus callosum showed no significant differences in the number of ASPA-positive oligodendrocytes, which may be related to the rapid endogenous remyelination that occurs in this region (Supplemental Figure 3, E and K). However, electron microscopy (EM) analysis showed an increase in the number of myelinated axons in the corpus callosum with both T3 and sobetirome treatment as compared with vehicle treatment (Figure 2, G and H). In addition, the g-ratios (the ratios of the inner axonal diameter to the total outer diameter) of the myelinated axons showed a shift toward higher ratios in all mice treated with cuprizone $(0.83 \pm 0.01$ for control, $0.84 \pm 0.01$ for $\mathrm{T} 3$, and $0.84 \pm 0.01$ for sobetirome, mean \pm SEM) as compared with naive mice $(0.78 \pm 0.01, P<0.001$ for all comparisons between naive and cuprizone conditions). No differences were observed between the g-ratios in treatment and control groups from cuprizone-administered mice (Supplemental Figure 4). 


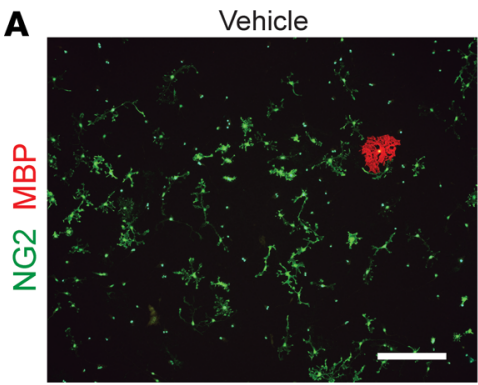

C

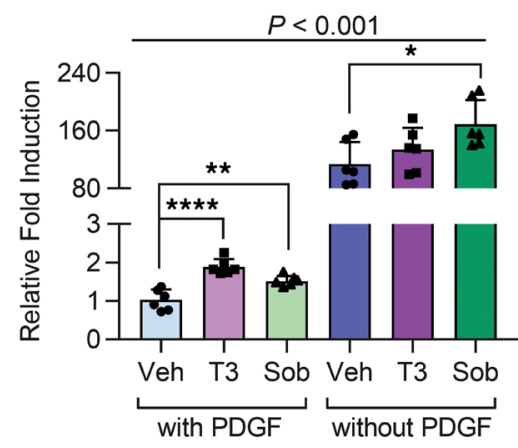

T3

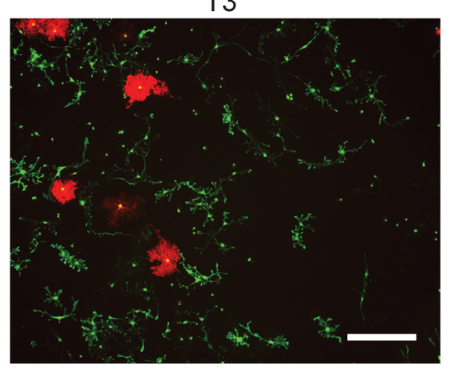

D

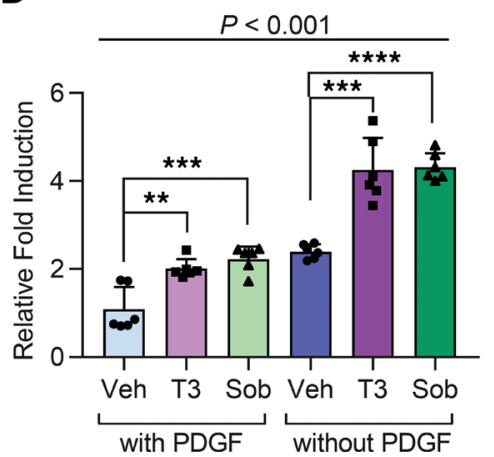

Sobetirome

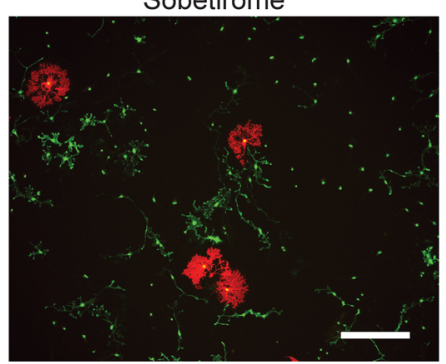

E

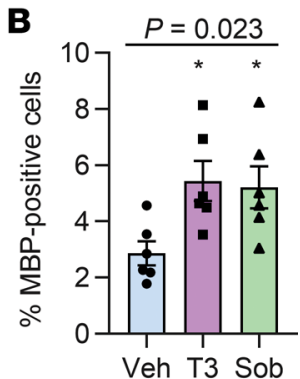

$H r$

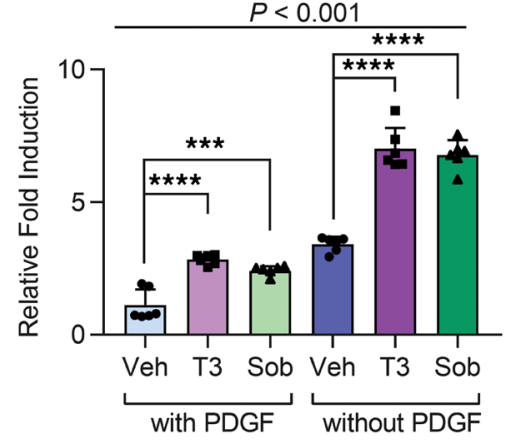

Figure 1. Sobetirome mimics thyroid hormone action in oligodendrocyte progenitor cells. (A) Oligodendrocyte progenitor cells (OPCs) were incubated with vehicle (DMSO), $50 \mathrm{nM} \mathrm{T3}$, or $50 \mathrm{nM}$ sobetirome for 96 hours in the presence of PDCF. Representative images of OPCs stained with antibodies against NG2 for OPCs (green) and MBP for oligodendrocytes (red). Scale bars: $200 \mu \mathrm{m}$. (B) Quantification of MBP-positive oligodendrocytes demonstrated that T3 or sobetirome increased OPC differentiation. (C-E) OPCs isolated from rats were incubated with or without PDGF in the presence of DMSO (Veh), T3 (50 $\mathrm{nM}$ ), or sobetirome (50 $\mathrm{nM}$ ) for 24 hours. Transcript levels of $\mathrm{Mbp}, \mathrm{KIfg}$, and $\mathrm{Hr}$ were measured using qPCR. OPC isolation was performed in triplicate with technical qPCR duplicates. Statistical significance was determined by 1-way ANOVA across all groups ( $P$ value in figure) followed by a 2-tailed, unpaired $t$ test for comparisons between groups denoted with asterisks ( $\left.{ }^{*} P \leq 0.05,{ }^{* *} P \leq 0.01,{ }^{* * *} P \leq 0.001,{ }^{* * * *} P \leq 0.0001\right)$. All graphs show mean \pm SEM.

iCKO-Myrf mice represent a demyelination model that correlates myelin pathology to motor disability. Both the cuprizone and lysolecithin models are characterized by rapid, spontaneous remyelination and lack robust and reproducible clinical signs that correlate with demyelination. We sought to test sobetirome in a model without these disadvantages. We chose a genetic mouse model in which demyelination is induced by tamoxifen treatment of adult Myrflff; Plp1-CreERT mice (32). Functional Myrf is essential for both the maintenance of myelin and the long-term survival of oligodendrocytes; thus, tamoxifen-induced Myrf ablation results in widespread CNS oligodendrocyte death and demyelination associated with clinical signs, including ataxia and locomotor deficit (32). We reasoned that this model would be particularly useful for evaluating remyelinating agents, because Myrf deletion only occurs in mature oligodendrocytes, which allows unaffected OPCs to repopulate the affected areas, differentiate to new oligodendrocytes, and remyelinate the CNS.

Demyelination in this strain was previously studied to establish the importance of Myrfin oligodendrocyte viability (32); however, the time course of remyelination and any corresponding improvement in clinical score was not thoroughly characterized. We began by assessing the natural history of the demyelinating disease brought on by transient tamoxifen treatment (once daily, 5 days, i.p.) of 8-week-old iCKO-Myrf mice. Myrf $f^{l / f l}$ mice not expressing Plp1-CreERT were used as controls throughout the experiment. Clinical signs of locomotor disability were quantified by weekly rotarod analysis and showed a nadir of rotarod latency 10-14 weeks after tamoxifen treatment, followed by performance recovery over 14-18 weeks, culminating in a stable plateau from 18-32 weeks, with reduced performance from baseline, representing irreversible disability (Figure 3A). As Myrf is not required for Schwann cell myelination in the peripheral nervous system $(32,33)$, these motor performance deficits presumably reflected the effects of CNS demyelination. BlackGold myelin staining of brains from week 10 showed little myelin staining (Figure 3B), confirming the extensive CNS-wide demyelination described previously in this model (32). At 24 weeks after tamoxifen induction, an increase in myelin staining corresponded with the observed partial motor recovery but did not reach the same level as control Cre-negative littermates (Figure 3B). Female mice performed better than male mice at rotarod, both before and after peak disease (Supplemental Figure 5A), suggesting 
A

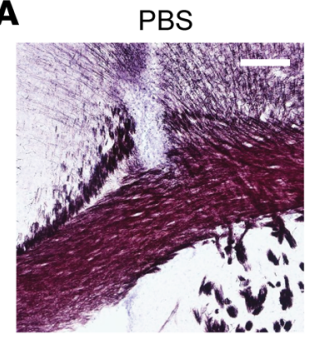

Lysolecithin

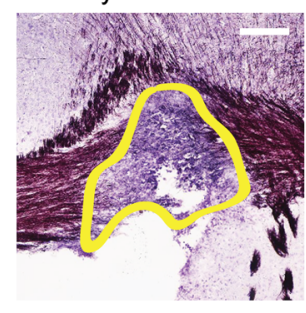

B

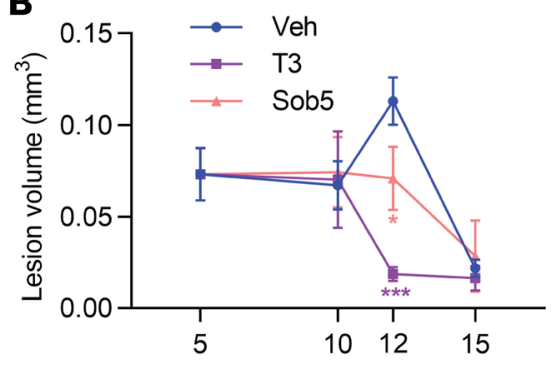

Days post-lysolecithin
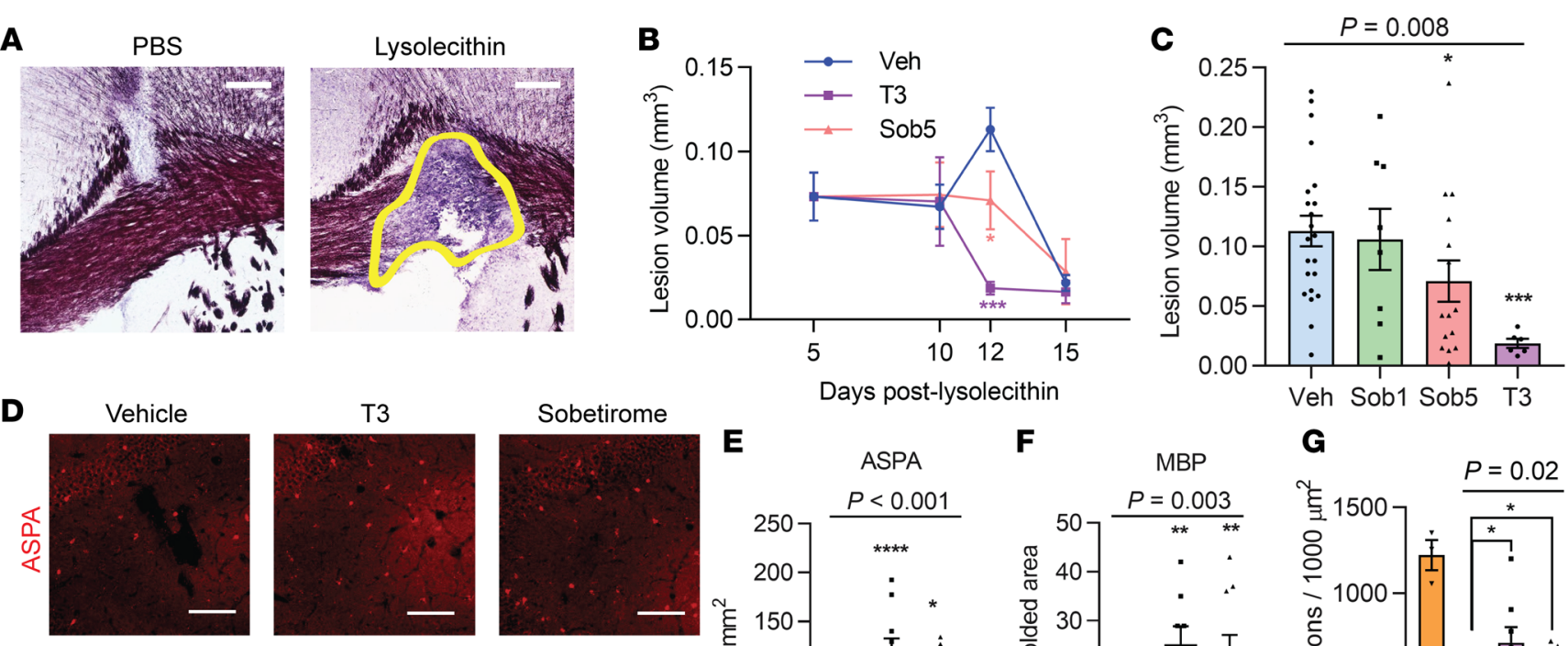

E

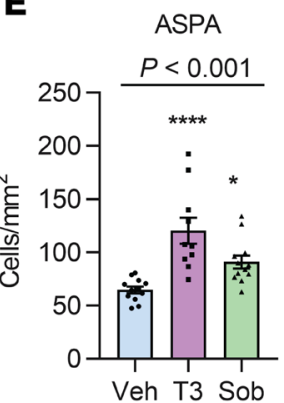

F
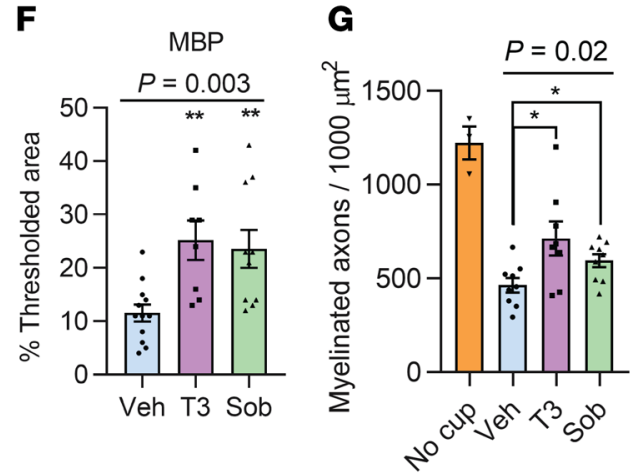

T3
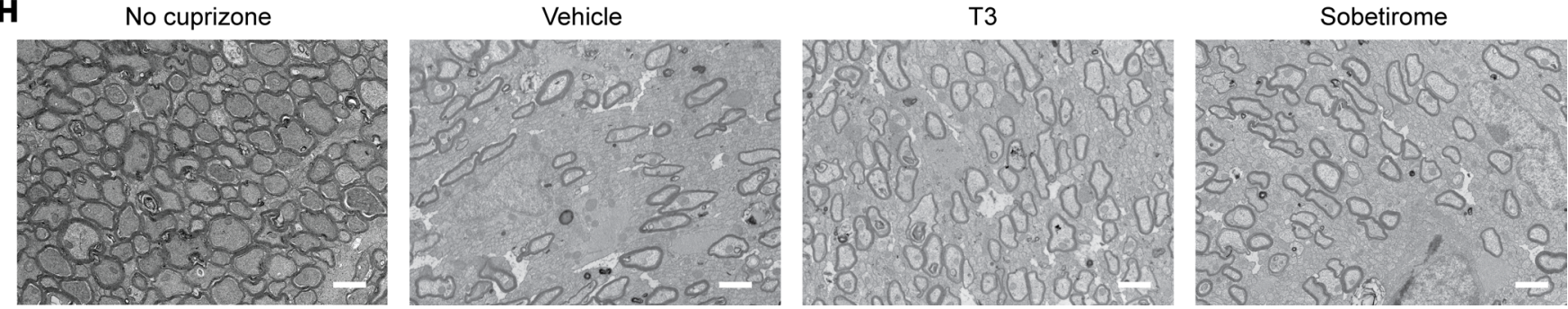

Figure 2. T3 and sobetirome treatment promotes myelin repair in lysolecithin and cuprizone models. (A) BlackGold staining of demyelinated lesion after PBS or lysolecithin injection (lesion outlined in yellow). Scale bars: $200 \mu \mathrm{m}$. (B and C) Mice were injected with lysolecithin on day 0, and daily i.p. injections with vehicle, T3 $(1 \mathrm{mg} / \mathrm{kg}$ ), or sobetirome (1 or $5 \mathrm{mg} / \mathrm{kg}$ ) began on day 5. (D-H) Mice were treated with cuprizone for 12 weeks followed by 3 weeks of normal chow and daily i.p. injections with vehicle, T3 $(1 \mathrm{mg} / \mathrm{kg})$, or sobetirome $(1 \mathrm{mg} / \mathrm{kg})$. (D) Representative immunofluorescence images in the hippocampus. Brain sections were stained with antibodies for ASPA (red) and MBP (green). Scale bars: $200 \mu \mathrm{m}$. (E and F) ASPA-positive cells were quantified, and MBP intensity was quantified by threshold analysis. Data represent the following groups (for ASPA, Veh [ $n=6]$, T3 [ $n=5]$, and Sob [ $n=6]$; for MBP, Veh $[n=6]$, T3 $[n=4]$, and Sob [ $n=5]$ ). Two images were quantified for each animal. (G) Quantification of myelinated axons in the corpus callosum. Data represent $n=3$, and 3 images were quantified for each animal. Naive mice that did not receive cuprizone (No cup) were not included in the statistical analysis. (H) Representative electron microscopy images from the medial caudal corpus callosum. Scale bars: $1 \mu \mathrm{m}$. Statistical significance was determined by 1-way ANOVA across all groups ( $P$ value in figure) followed by a 2-tailed, unpaired $t$ test for comparisons between vehicle and treatment denoted with asterisks $\left({ }^{*} P \leq 0.05,{ }^{* *} P \leq 0.01,{ }^{* * *} P \leq 0.001,{ }^{* * * *} P \leq 0.0001\right)$. All graphs show mean $\pm \mathrm{SEM}$.

that this difference is unrelated to the demyelinating phenotype. There were no differences in overall brain myelin staining between the sexes at 24 weeks (Supplemental Figure 5, B and C), further supporting the conclusion that there are minimal sex differences in demyelination in this model. All subsequent experiments were performed with an even distribution of male and female mice.

We have further characterized the disease time course in these mice by in vivo whole-brain MRI using magnetization transfer ratio (MTR) imaging, a semiquantitative technique sensitive to myelin. MTR maps acquired in iCKO-Myrf mice at 10 and 24 weeks correlate with the histological findings: decreased MTR in the corpus callosum (iso-intense with adjacent gray matter) at week 10 and limited recovery of MTR (hyperintense compared with adjacent gray matter) at week 24 as compared with that in wild-type mice (Figure 3C). Comparison of MTR values across the corpus callosum showed increases in MTR between week 10 and 24 (Supplemental Figure 6, C and D) but much lower levels than in wild-type mice (34). Thus, the iCKO-Myrf model is characterized by the slow onset of CNS-wide demyelination that tracks with increasing severity of clinical signs followed by a 4 -week recovery phase and stabilization with permanent 
A

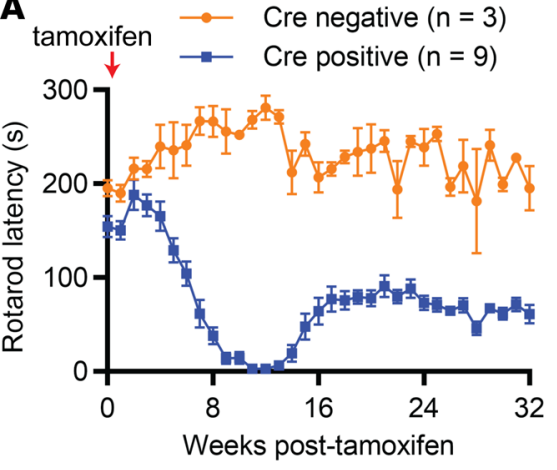

B Cre negative

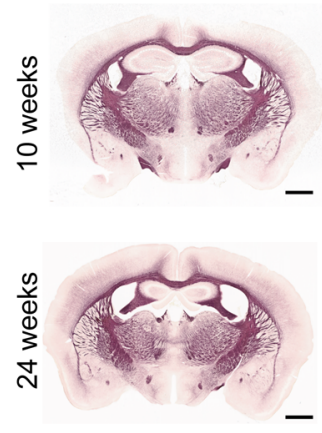

Cre positive

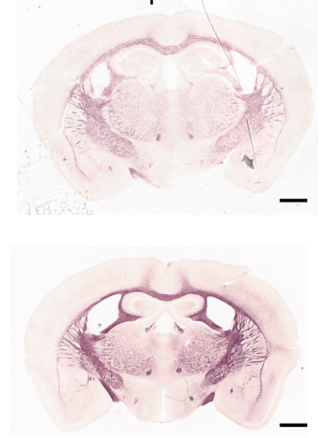

C

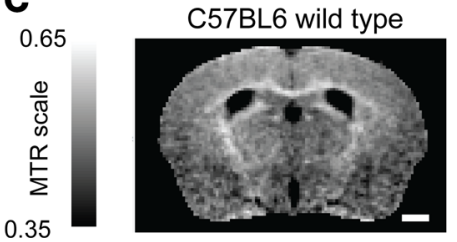

Cre positive 10 weeks

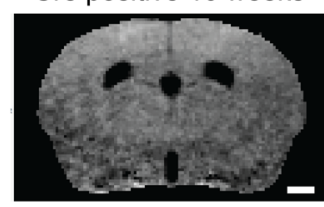

Cre positive 24 weeks

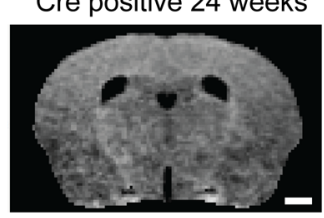

Figure 3. After Myrf ablation, iCKO-Myrf mice experience clinical signs of demyelination and remyelination consistent with histology findings. (A) Myrf ablation was induced at 8 weeks of age in iCKO-Myrf mice with daily i.p. doses of tamoxifen over 5 days. Mice performed the rotarod test weekly for 32 weeks after tamoxifen injection. (B) Representative BlackGold images of Cre-positive brains at week 10 and week 24 compared with Cre-negative controls. Scale bars: $1 \mathrm{~mm}$. (C) Serial, semiquantitative magnetization transfer ratio (MTR) maps at week 10 and week 24 (right) averaged from 3 mice. Averaged MTR map from wild-type C57BL6 mice (left, $n=5$ ) was adapted with permission from PLoS ONE (34). Note the hyperintense myelinated white matter tracts in the wild-type mice, compared with isointense and slightly hyperintense corresponding areas in the week 10 and week 24 Cre-positive mice, respectively. Scale bars: $1 \mathrm{~mm}$.

locomotor disability. CNS myelin content detected by both histology and MRI changes through this disease progression in a manner consistent with the behavioral profile.

Sobetirome hastens motor recovery and myelin repair in iCKO-Myrf mice. To evaluate the effects of sobetirome, iCKO-Myrf mice were provided ad lib access to chow containing sobetirome starting at 2 weeks after tamoxifen induction for the remaining 22-week duration of the experiment. Oral dosing with sobetirome-fortified chow in a previous study resulted in the predicted systemic drug exposure and was effective for delivering sobetirome to the CNS and inducing thyromimetic action (24). The dose of sobetirome (nominal $80 \mu \mathrm{g} / \mathrm{kg} / \mathrm{d}$ ) was empirically determined previously to be the highest tolerated dose of chowadministered sobetirome (24). A clinical scoring system was used to assess motor disability (32), and mice receiving sobetirome had a faster improvement in clinical scores relative to the control mice (Figure 4A). Weekly rotarod analysis showed that sobetirome-treated mice recovered sooner (Figure 4, B and C) and had a significant increase in the extent of functional motor recovery compared with that of control mice (Figure 4D). In contrast, sobetirome treatment did not appear to affect the rate of performance decline, suggesting that the demyelination course occurs independently from treatment effects.

Further, this motor improvement correlated to myelin levels. BlackGold staining was performed to assess overall myelin levels, and threshold analyses were performed to assess both white and gray matter myelin (Supplemental Figure 7A). With a limited threshold that included only intense myelin staining in white matter tracts, significantly more myelin was observed at 24 weeks with sobetirome treatment (Figure $5, \mathrm{~A}-\mathrm{D})$. A second threshold analysis was performed to assess gray matter myelin, and significantly more cortical and gray matter myelin was also observed in the rostral brain regions (Supplemental Figure 7B, $P \leq 0.05)$ with sobetirome treatment. In addition, spinal cords from mice treated with sobetirome showed a greater proportion of myelinated axons at 24 weeks after tamoxifen indicative of a greater extent of myelin repair (Figure 5, E and F). Remyelinated axons typically display thinner myelin than developmentally myelinated axons (35). G-ratio analysis (Figure 5G) confirmed that the vast majority of the axons observed at 24 weeks had thinner myelin $(0.80 \pm 0.01$ for control and $0.80 \pm 0.01$ for sobetirome, mean \pm SEM) as compared with Cre-negative controls $(0.73 \pm 0.01, P<0.001$ for all comparisons between Cre-negative and Cre-positive conditions). No difference was observed in the g-ratios between controland sobetirome-treated mice, consistent with the cuprizone data (Supplemental Figure 4) and supporting 

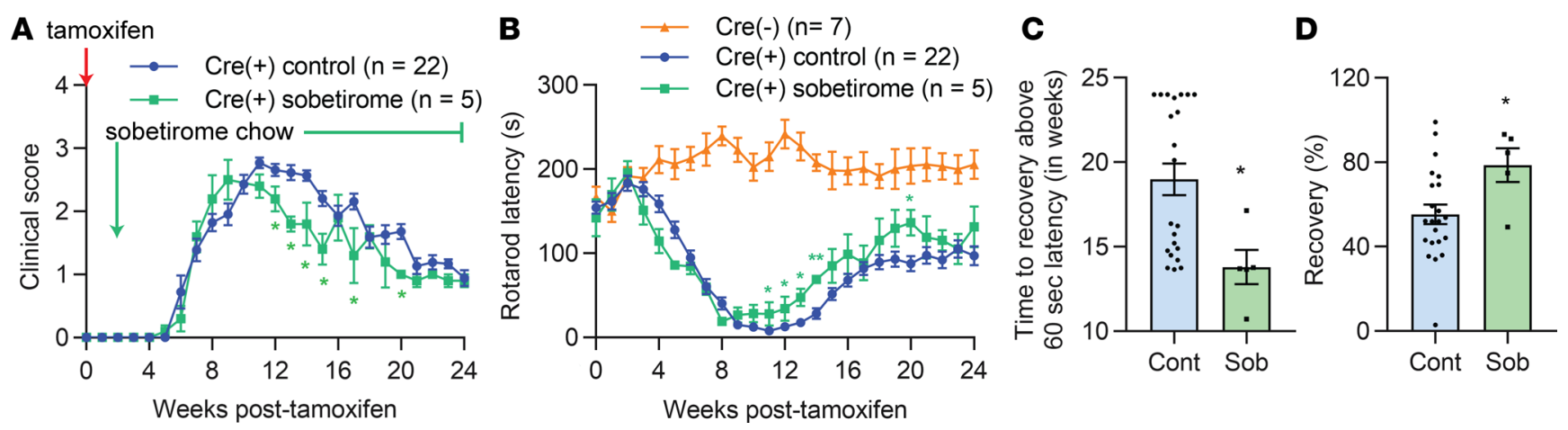

Figure 4. Treatment with sobetirome improves motor performance in iCKO-Myrf mice. iCKO-Myrf mice were induced with 5 days of once-daily tamoxifen i.p. injections, and starting 2 weeks later, a cohort was fed ad lib chow compounded with sobetirome ( $80 \mu \mathrm{g} / \mathrm{kg} / \mathrm{d}$ nominal dose). The control cohort was provided normal chow. (A) Clinical scores were assigned weekly (0, no disability; 1 , wide gait or limp tail; 2 , hindlimb weakness; 3 , hindlimb paralysis). (B) Rotarod testing was performed weekly. (C) Summary analysis of rotarod data was performed by determining the week at which the mice crossed above a 60-second threshold for the remainder of the experiment. (D) Individual recovery was measured by dividing the average latency during the recovery phase (weeks 18-24) by the latency before decline (weeks $0-4$ ). Statistical significance was determined by a 2-tailed, unpaired $t$ test comparing control to sobetirome ( $\left.{ }^{*} P \leq 0.05,{ }^{* *} P \leq 0.01\right)$. In $\mathbf{A}$ and $\mathbf{B}$, each $t$ test was performed independently. All graphs show mean \pm SEM.

the conclusion that sobetirome-mediated myelin repair does not increase myelin thickness in the cuprizone or iCKO-Myrf models.

$\mathrm{TH}$ action in the CNS is known to promote the differentiation of OPCs into myelinating oligodendrocytes both during development and remyelination (18). To establish whether sobetirome promotes myelin repair via enhanced differentiation, iCKO-Myrf mice treated with control or sobetirome chow were administered drinking water containing 5-ethynyl-2'-deoxyuridine (EdU) to label proliferating OPCs starting 4 weeks after tamoxifen induction. Mice were euthanized at week 10, at which time the formation of new oligodendrocytes has begun, but this is prior to remyelination (32). Sobetirome treatment significantly increased the number of newly formed EdU-labeled oligodendrocytes in the caudal corpus callosum, without depleting the OPC population (Figure 5, H-J). This result supports the proposed mechanism that sobetirome promotes OPC maturation into oligodendrocytes.

Evaluation of thyroid status in iCKO-Myrf mice reveals a selective thyromimetic mechanism of sobetirome. Since sobetirome is a selective T3 agonist $(19,36)$, it was important to establish functional effects of thyroid status in the iCKO-Myrf model. Either hypo- or hyperthyroidism was induced in iCKO-Myrf mice using standard pharmacological methods (37) 2 weeks after tamoxifen injections, and the altered thyroid status was maintained until the conclusion of the experiment. Hypothyroidism was confirmed by T4 radioimmunoassay $(6.5 \pm 0.3 \mu \mathrm{g} / \mathrm{d}$ for controls and $0.5 \pm 0.2 \mu \mathrm{g} / \mathrm{dl}$ for hypothyroid, mean $\pm \mathrm{SEM})$. Chronic hyperthyroidism exacerbated the motor deficits of the iCKO-Myrfmice, and this cohort was euthanized 14 weeks after tamoxifen, as they displayed a more severe clinical course, including ataxia and no improvement in rotarod latency (Figure 6A). Cre-negative iCKO-Myrf mice on T3/T4 chow also showed decreased performance on rotarod (Supplemental Figure 8A), which suggests that the motor deficits were due in part to systemic thyrotoxicosis, but this partial performance decrease does not fully account for the observation that T3/T4-treated Cre-positive iCKO-Myrf mice showed no signs of recovery between 10 and 14 weeks. In contrast, chronic hypothyroidism had no significant effects on rotarod performance or remyelination. Hypothyroid mice showed similar recovery to control mice (Figure 6, B and C), similar myelin staining in the white matter tracts (Figure 6, D-G), and significantly reduced brain myelin content in cortical regions (Supplemental Figure 7C, $P \leq 0.05$ ).

To determine if the effects of chronic hyper- and hypothyroidism exerted a central effect on remyelination, a second cohort of tamoxifen-induced iCKO-Myrf mice was rendered hyper- and hypothyroid and cotreated with EdU at 4 weeks after tamoxifen induction. At 10 weeks, we observed that PDGFR $\alpha / E d U$ OPCs and ASPA/EdU-positive oligodendrocytes were significantly reduced in hyperthyroid mice relative to mice receiving control chow, whereas the levels in hypothyroid mice were unaffected (Figure 7). These data indicate that chronic hyperthyroidism inhibited the production of new OPCs and oligodendrocytes, whereas chronic hypothyroidism had no effect on these glial populations. These results further underscore the need for selective $\mathrm{TH}$ action, such as that provided by sobetirome, to promote remyelination in the CNS. 
A Control

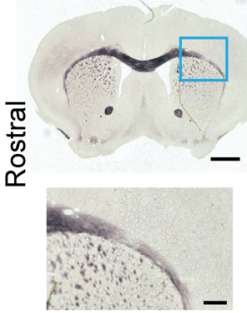

E

Control
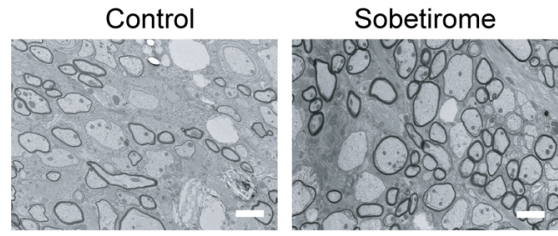

H
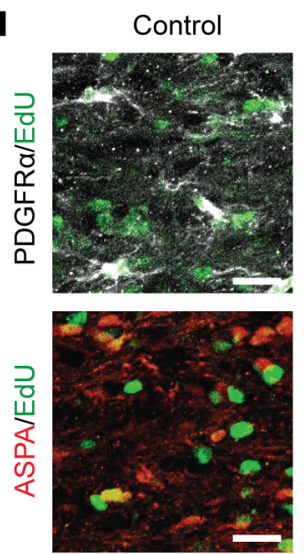

Sobetirome
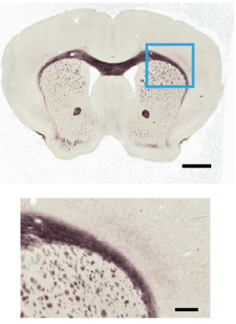

Sobetirome

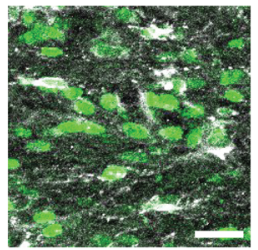

B Control

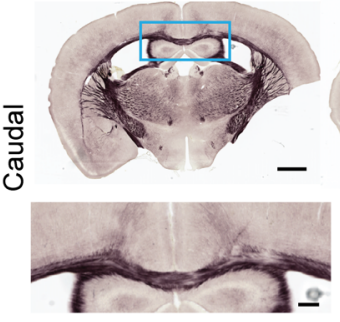

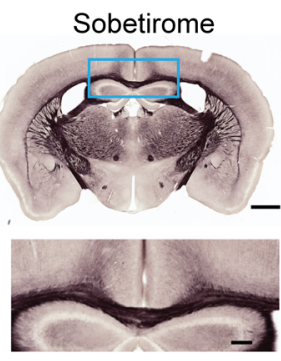

F

Cre negative
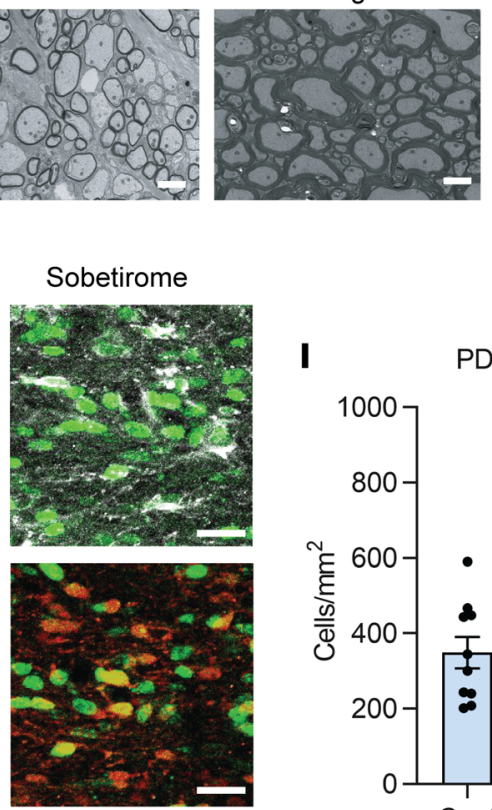

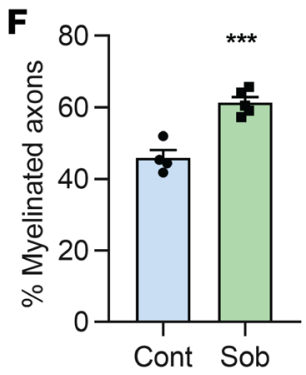

Cont Sob
C Rostral D

D Caudal

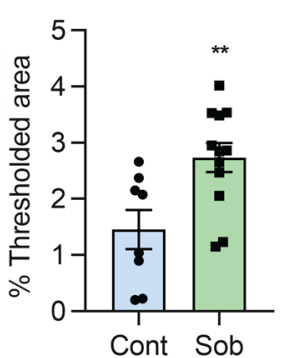

$\mathbf{G}_{1.0}$

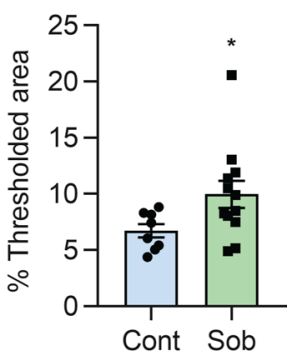

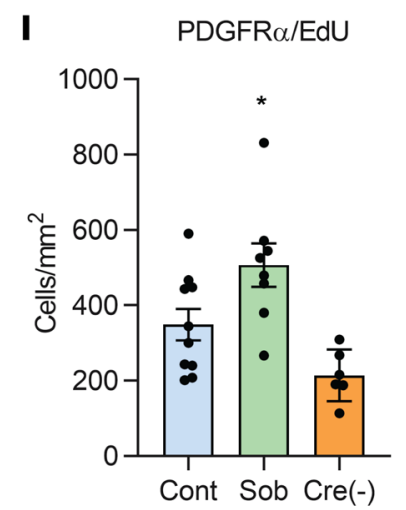

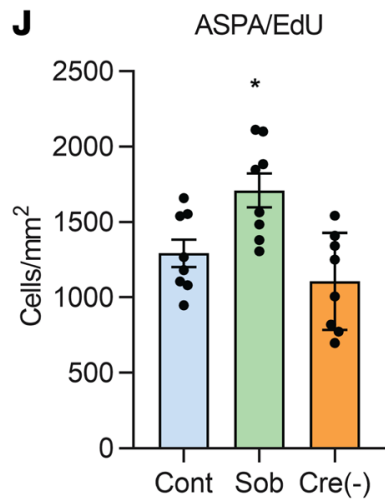

Figure 5. Sobetirome hastens myelin recovery and oligodendrocyte maturation in iCKO-Myrf mice. (A and B) Representative BlackGold images from Cre-positive mice treated with control or sobetirome chow. Scale bars: $1 \mathrm{~mm}$ (full-brain images); $0.25 \mathrm{~mm}$ (magnified inset, blue box). (C and D) Quantification of BlackGold images was performed by threshold analysis for white matter tracts ( $n=4$ for control and $n=6$ for Sob, 2 images per animal). (E) Representative spinal cord EM images from control- and sobetirome-treated Cre-positive mice and Cre-negative mice at 24 weeks. Scale bars: $2 \mu \mathrm{m}$. (F) Myelinated axons were quantified in the ventrolateral spinal cord ( $n=4$ for control, $n=5$ for Sob, 2 images per animal). (G) G-ratio analysis of spinal cord axons representing 100-200 axons per group. (H) Representative images from the caudal corpus callosum at week 10 stained for PDGFR (white), ASPA (red), and EdU (green) from mice treated with control or sobetirome chow from weeks 2-10 and coadministered EdU from weeks 4-10. Scale bars: $20 \mu \mathrm{m}$. (I and J) Quantification of the PDGFR $\alpha /$ EdU colabeled oligodendrocyte precursor cells (OPCs) and ASPA/EdU-colabeled oligodendrocytes ( $n=4$ for all groups, 2 images per animal). Statistical significance was determined by a 2 -tailed, unpaired $t$ test comparing control to sobetirome $\left({ }^{*} P \leq 0.05\right.$, $\left.{ }^{* *} P \leq 0.01,{ }^{* *} P \leq 0.001\right)$. All graphs show mean $\pm \mathrm{SEM}$.

A CNS-penetrating prodrug derivative of sobetirome, Sob-AM2, improves motor and myelin recovery in iCKOMyrf mice. To improve the CNS penetration of sobetirome, a prodrug strategy has been developed recently $(20,21,38)$. Sob-AM2, a methyl amide derivative of sobetirome, is an optimized prodrug that significantly increases delivery of sobetirome to the brain while at the same time decreasing the sobetirome blood and peripheral organ exposure (38). We sought to evaluate the effect of Sob-AM2 treatment in the iCKO-Myrf model. We treated mice with a dose of Sob-AM2 in chow equivalent to the sobetirome experiment ( $84 \mu \mathrm{g} / \mathrm{kg} / \mathrm{d}$, p.o.) starting at 2 weeks after tamoxifen. Weekly rotarod analysis showed a significant benefit of Sob-AM2 treatment over control mice, with faster and more robust recovery (Figure 8, A-C). During most of the recovery and plateau period (weeks 10-22), Sob-AM2treated mice showed significantly increased rotarod latencies versus control (Figure 8A and Supplemental Figure $8 \mathrm{C}$ for direct comparison with sobetirome). Mice treated with Sob-AM2 showed no statistical difference in performance from control Cre-negative mice during 3 separate weeks during the 
A

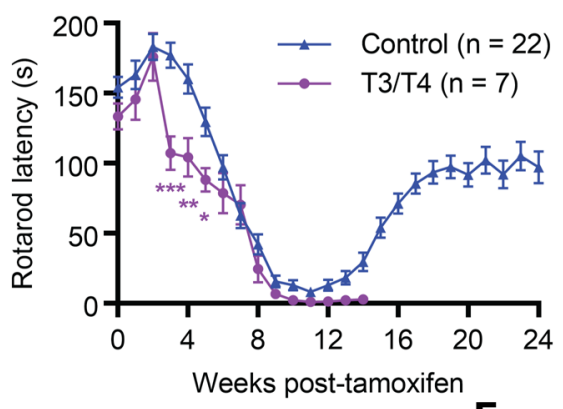

B

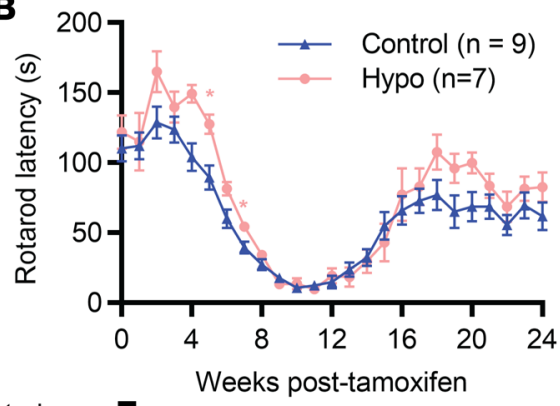

C

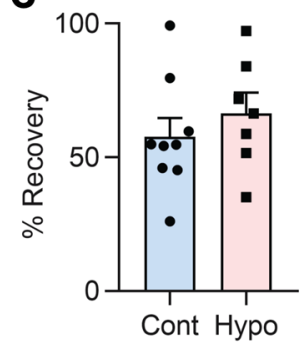

D

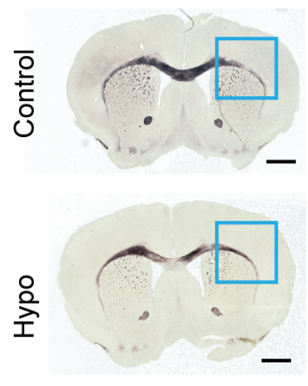

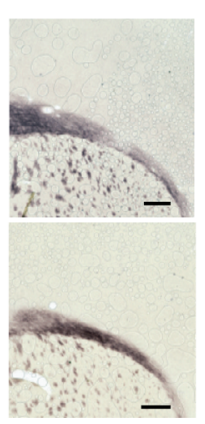
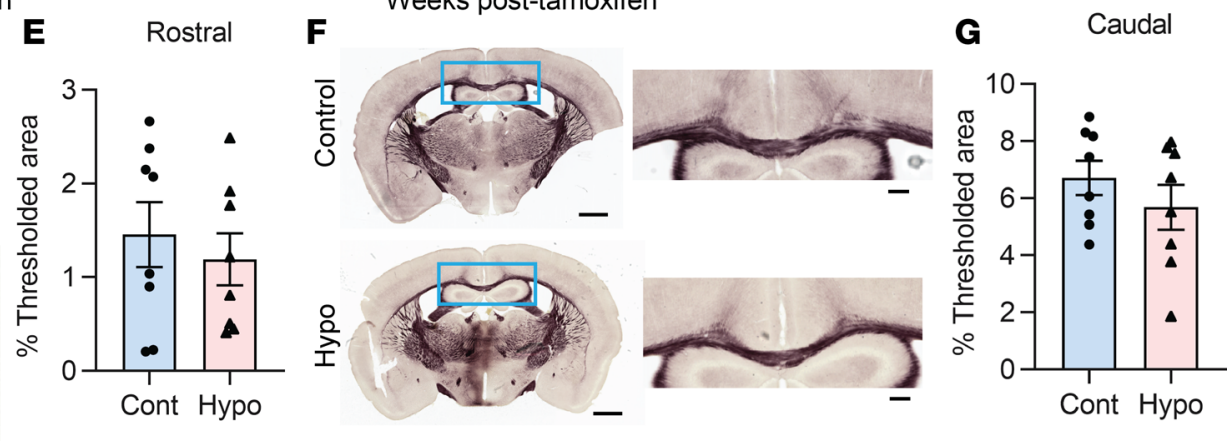

Figure 6. Hyper- and hypothyroidism produce different outcomes in the iCKO-Myrf model. (A) Mice were administered T3/T4 chow starting 2 weeks after tamoxifen. The mice experienced a more severe disease course with no recovery and were euthanized at 14 weeks. (B) Mice were administered hypothyroid-inducing water starting 2 weeks after tamoxifen and were followed weekly by rotarod analysis. (C) Individual recovery was measured by dividing the average latency during the recovery phase (weeks 18-24) by the latency before decline (weeks 0-4). ( $\mathbf{D}$ and $\mathbf{F}$ ) Representative BlackGold brain images from control and hypothyroid groups. Scale bars: $1 \mathrm{~mm}$ (full-brain images); $0.25 \mathrm{~mm}$ (magnified insets, blue box). (E and G) Quantification of BlackGold images was performed by threshold analysis for white matter tracts $(n=4,2$ images per animal). Statistical significance was determined by a 2-tailed, unpaired $t$ test comparing control to treatment $\left({ }^{*} P \leq 0.05\right)$. In $\mathbf{A}$ and $\mathbf{B}$, each $t$ test was performed independently $\left({ }^{*} P \leq 0.05,{ }^{* *} P \leq 0.01,{ }^{* * *} P \leq 0.001\right)$. No differences were significant for $\mathbf{C}\left({ }^{*} P=0.42\right), \mathbf{E}\left({ }^{*} P=0.56\right)$, or $\mathbf{G}\left({ }^{*} P=0.31\right)$. All graphs show mean $\pm \mathrm{SEM}$.

plateau stage (weeks 19,21 , and $23 ; P>0.10$ ), suggesting a return to baseline performance that was not observed with sobetirome. These results are corroborated by the recovery levels of Sob-AM2 mice (Figure 8C, 95\% $\pm 13 \%$, mean \pm SEM), which were higher than those of mice treated with sobetirome (Figure $4 \mathrm{D}, 79 \% \pm 8 \%$ ) and significantly higher than those of controls (Figure $8 \mathrm{C}, 55 \% \pm 5 \%$ ). Finally, comparison of the rotarod performance of Cre-negative mice treated with sobetirome or Sob-AM2 (Supplemental Figure 8B) suggested that Sob-AM2 treatment in healthy mice had minimal effects on rotarod performance compared with sobetirome, which may arise from the lower peripheral sobetirome exposure afforded by the prodrug Sob-AM2 (38).

The improvements in performance with Sob-AM2 were correlated with increased BlackGold myelin staining both in white matter tracts (Figure 8, D, E, and G) and in gray matter regions (Supplemental Figure 7D). MTR data were acquired on a small subset of the control and Sob-AM2 cohorts ( $n=3$ for each) at weeks 10, 15, and 24 (Figure 8F and Supplemental Figure 6, A and B). At week 10, both control and Sob-AM2 mice showed iso-intense MTR throughout the corpus callosum consistent with extensive loss of myelin (Supplemental Figure 6A), and little change was observed at week 15 (Supplemental Figure 6, B and D-F). At week 24, however, mice treated with Sob-AM2 showed higher MTR values in the body/isthmus of corpus callosum (Figure 8F, white arrows) and in lateral corpus callosum regions (Figure $8 \mathrm{~F}$, white arrowheads) relative to the same regions in control untreated mice (Figure 8F, yellow arrows and arrowheads). Relative recovery in this region can be quantified by $\triangle$ MTR, the difference between the lowest MTR at week 10 or 15 and that at week 24 (Supplemental Table 1). The $\triangle$ MTR in Sob-AM2-treated mice was twice that in untreated controls ( $0.048 \pm 0.001 \mathrm{vs}$. $0.022 \pm 0.006$, mean \pm SEM, $P=0.02$ ). In addition, comparison of the MTR values along the corpus callosum showed similar demyelination at weeks 10 and 15 for both groups and delineated a treatment effect for Sob-AM2 over control at week 24 (Supplemental Figure 6, C and G). Finally, the BlackGold myelin staining of the mice imaged with MRI (highlighted in Figure 8E with orange data points) tracked with the improvement observed in MTR and was representative of the larger cohort of control and Sob-AM2-treated mice. 
A
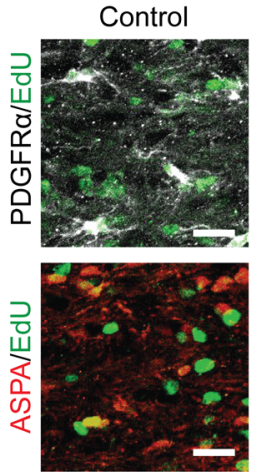

B

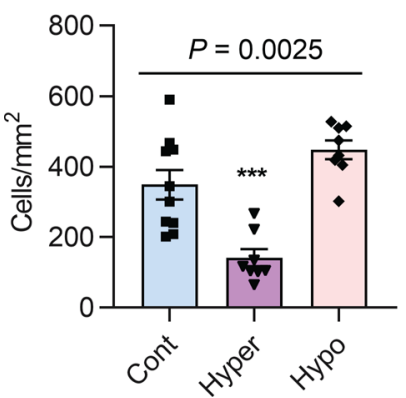

Hyperthyroid

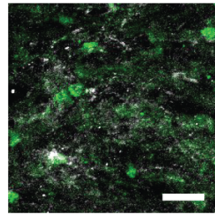

Hypothyroid
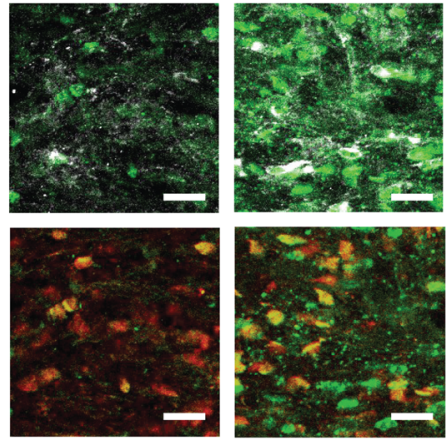

C

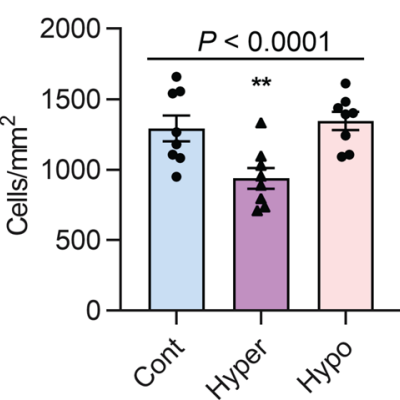

ASPAVdU

Figure 7. Hyperthyroid treatment in iСКО-Myrf mice reduces oligodendrocyte progenitor cell and oligodendrocyte populations. (A) Representative images from the caudal corpus callosum at week 10 stained for PDCFR $\alpha$ (white), ASPA (red), and EdU (green) from mice treated with control chow, T3/T4 chow, or hypothyroid water from weeks 2-10 and coadministered EdU from weeks 4-10. Scale bars: $20 \mu \mathrm{m}$. (B and C) Quantification of the PDGFR $\alpha /$ EdU colabeled oligodendrocyte progenitor cells (OPCs) and ASPA/EdU colabeled oligodendrocytes ( $n=4,2$ images per animal). Statistical significance was determined by ANOVA analysis with Dunnett's multiple comparisons test comparing control to treatment groups $\left({ }^{* *} P \leq 0.01,{ }^{* * *} P \leq 0.001\right)$. All graphs show mean $\pm \mathrm{SEM}$.

\section{Discussion}

CNS-selective thyromimetics promote myelin repair in a chronic model. No clinically approved therapies are available that promote myelin repair, which is a proposed goal for preventing long-term neurodegeneration in MS and other demyelinating diseases $(35,39)$. Two primary strategies for achieving this goal involve the removal of inhibitors preventing remyelination or identifying molecules that promote OPC proliferation and differentiation (35). The former strategy is best exemplified by the development of a monoclonal antibody that targeted LINGO1 (opicinumab), an integral membrane protein that inhibits OPC differentiation. Treatment with opicinumab for 6 months showed modest but borderline significant improvements in visual evoked potential of patients with acute optic neuritis; however, anti-LINGO1 treatment failed to show significant improvement in disability in a phase 2 clinical trial in MS patients (35). Small-molecule approaches to date have primarily focused on the second strategy, with high-throughput screening of both approved drug collections and large small-molecule libraries to identify drug candidates that increase OPC differentiation (8-10). Screening hits have been evaluated in traditional demyelination rodent models and mechanisms of action that may explain pro-remyelinating properties have been identified post-hoc $(40,41)$. This approach ultimately hinges on the ability to repurpose an approved drug for a demyelination indication or enter into clinical development with a remyelination mechanism of action that is novel or not fully understood. Issues, such as CNS penetration and undesired or incompatible peripheral side effects, can present significant problems for this approach.

In contrast, sobetirome is a clinical stage experimental thyromimetic therapy that can access the CNS to exert transcriptional and biochemical effects $(22,24)$ via agonist action at TRs $(19)$, which are known to be involved in myelination $(3,7)$. Sob-AM2 is a novel prodrug of sobetirome that when dosed systemically leads to increased CNS distribution and decreased peripheral exposure of the parent drug sobetirome. Sob-AM2 is selectively converted to sobetirome by fatty acid amide hydrolase (FAAH), which is highly expressed in the CNS (38). We observed significant improvements in rotarod performance and recovery in 


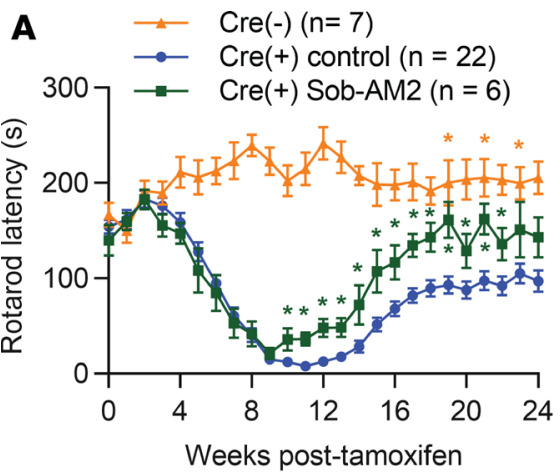

F
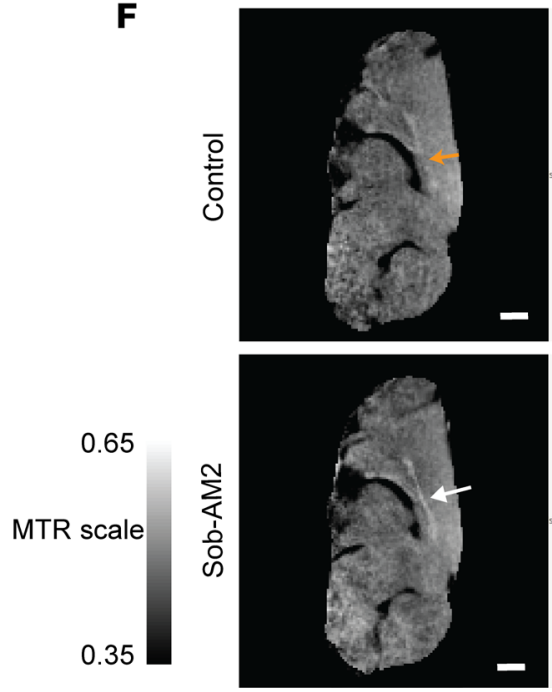

B
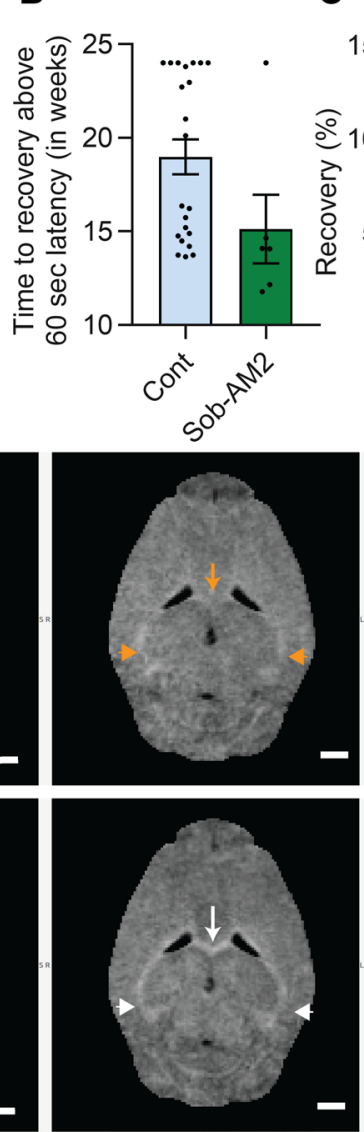

C
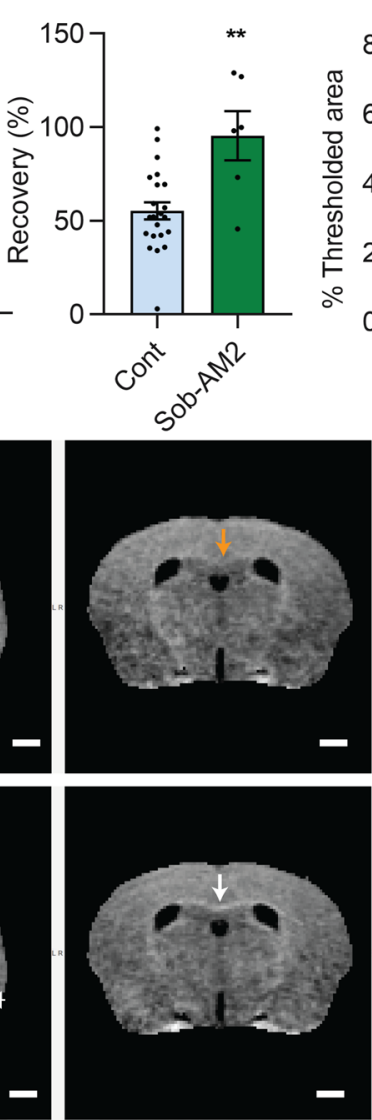

D

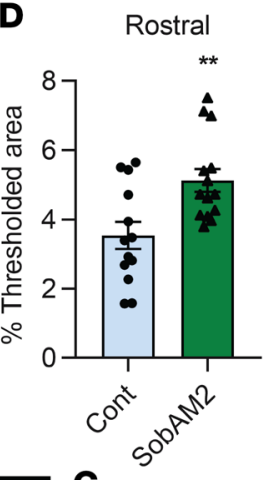

$\mathbf{E}$

G
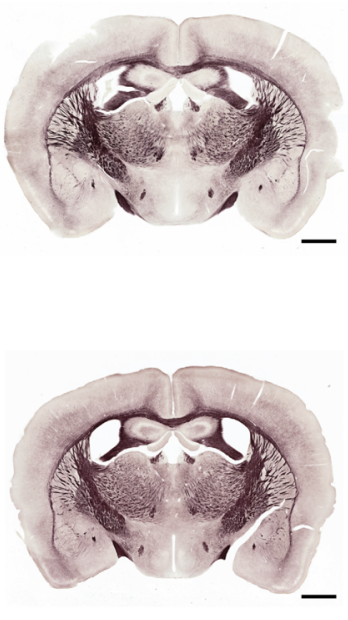

Caudal

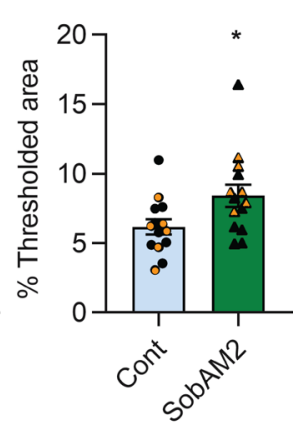

Figure 8. Sob-AM2 treatment in iCKO-Myrf mice improves motor performance and increases myelin recovery, as observed by MRI analysis. Ablation of Myrf was induced in iCKO-Myrf mice with 5 days of once-daily tamoxifen i.p. injections, and starting 2 weeks later, mice were fed ad lib chow compounded with Sob-AM2 ( $84 \mu \mathrm{g} / \mathrm{kg} / \mathrm{d}$ ) or control chow. (A) Rotarod testing was performed weekly. (B) Summary analysis of rotarod data was performed by determining the week at which the mice crossed above a 60 -second threshold for the remainder of the experiment. (C) Recovery was measured by dividing the average latency during the recovery phase (weeks 18-24) by the latency before decline (weeks 0-4). (D and E) Quantification of BlackGold images was performed by threshold analysis for white matter tracts ( $n=7,2$ images per animal). Data points marked with orange indicate mice imaged by MRI ( $n=3,2 \mathrm{images}$ per animal). (F) Group-average magnetization transfer ratio (MTR) maps from control and Sob-AM2 mice $(n=3)$ at week 24 . Arrows indicate the body/isthmus of the corpus callosum, and arrowheads indicate lateral corpus callosum (orange for weak remyelination and white for strong remyelination). Scale bars: 1 $\mathrm{mm}$. (C) BlackGold caudal brain images from representative control and Sob-AM2 mice imaged by MRI. Scale bars: 1 mm. Statistical significance was determined by a 2-tailed, unpaired $t$ test comparing control to Sob-AM2 ( ${ }^{*} P \leq 0.05,{ }^{* *} P \leq 0.01$ ). In A, each $t$ test was performed independently; green asterisks indicate weeks in which Sob-AM2 mice were significantly different from control Cre-positive mice $\left({ }^{*} P \leq 0.05\right)$, and orange asterisks indicate weeks in which the Sob-AM2-treated mice were not significantly different from unaffected Cre-negative mice $\left.{ }^{*} P>0.10\right)$. All graphs show mean \pm SEM.

the iCKO-Myrf model (Figures 4 and 8). Although Sob-AM2 treatment increases sobetirome CNS exposure compared with an equivalent dose of sobetirome by virtue of FAAH activity in the CNS, we have recently found that the magnitude of this distribution depends on the route of administration (42). In addition to being expressed in the CNS, FAAH is also present at high levels in the gastrointestinal tract, especially in rodents (43). Oral administration of Sob-AM2 used in the iCKO-Myrf model, therefore, leads to significant gastrointestinal conversion to sobetirome, which increases sobetirome and decreases Sob-AM2 concentration in the blood. In addition to this first-pass metabolic processing, the oral bioavailability of Sob-AM2 is about 5 -fold lower than that of sobetirome. The combination of these two issues results in a modest $(\sim 2$ - to 4-fold) increase in CNS sobetirome exposure provided by orally dosed Sob-AM2 compared with the same oral dose of sobetirome $(20,42)$. This is consistent with the modest improvement in rotarod performance we observed in iCKO-Myrf mice treated with $84 \mu \mathrm{g} / \mathrm{kg} / \mathrm{d}$ (p.o.) Sob-AM2 compared with mice treated with an equivalent sobetirome dose (Supplemental Figure 8C).

The clinical benefits of thyromimetics arise from a combination of selectivity factors that result in a therapeutic window that is not observed with TH treatment. First, sobetirome has approximately 10 -fold selectivity for the TR $\beta$ isoform over TR $\alpha$ (19). Many cell types in the CNS including OPCs primarily 
express $\mathrm{TR} \alpha$ (44), and analysis of $\mathrm{TR} \alpha-\mathrm{KO}$ mice suggested that $\mathrm{TR} \alpha$ was important for OPC differentiation during development (3). However, it has also been observed that TR $\alpha$ is primarily expressed in OPCs during neurodevelopmental stages associated with proliferation and that TR $\beta$ levels increase during later phases associated with OPC differentiation (7). This latter result is consistent with our finding that TR $\beta$-selective agonists promote OPC differentiation. A second factor is differential brain distribution of sobetirome and TH. TH is actively transported from blood to the CNS by MCT8 (45), whereas the mechanisms of sobetirome and Sob-AM2 CNS distribution are unknown (46) and, like other CNS drugs, may involve passive diffusion. These CNS uptake differences lead to zonal exposure differences within the brain and CNS that could affect the treatment outcome (38). A final contributing factor stems from the recent finding that sobetirome and Sob-AM2 act on the hypothalamic-pituitary-thyroid axis to induce central hypothyroidism (36). Thyromimetics act as agonists to suppress TSH production and induce systemic TH depletion. This unique endocrine status comprising low endogenous $\mathrm{TH}$ combined with supplementary thyromimetic exposure may promote oligodendrocyte maturation without adversely affecting OPC proliferation. This mechanism is consistent with our data showing a beneficial effect on both the OPC and oligodendrocyte populations with sobetirome treatment (Figure 5, H-J). While our data demonstrate that thyromimetics increase OPC and oligodendrocyte production in the iCKO-Myrf model, they do not preclude a role for thyromimetic action on other CNS cell types, including astrocytes, which have been implicated in TH-mediated remyelination in the cuprizone model (47). The therapeutically beneficial effects of sobetirome likely result from a combination of many factors underscoring the utility of selective thyromimetic action.

TH promotes myelin repair in short-term demyelination models, but has adverse effects in a chronic model. Our data from lysolecithin and cuprizone (Figure 2), as well as available literature on TH in remyelination models (11-17), suggest that short-term treatment with TH can promote the OPC-to-oligodendrocyte transition. In contrast, administration of chow containing $\mathrm{TH}(\mathrm{T} 3 / \mathrm{T} 4)$ was detrimental to recovery, with remarkable adverse clinical signs progressing with treatment and disease course (Figure 6). A key difference between the iCKO-Myrf model and traditional demyelination models is the duration of the disease state, which affects whether the TH administration is short term or chronic. In both the cuprizone and lysolecithin models, in which the treatment period is only 1-3 weeks, TH outperforms sobetirome as a remyelination agent. This likely stems from the fact that the TH treatment in the lysolecithin and cuprizone models is not a sufficient length to progress to the severe central and peripheral effects from thyrotoxicosis that develop in the longer treatment duration of the iCKO-Myrf model. In addition, TH has better brain penetration than sobetirome from systemic dosing and is more potent than sobetirome at TRs (48). These factors correlate with the better remyelination effects observed for short-term TH treatment in the lysolecithin and cuprizone models.

This stands in contrast to the iCKO-Myrf model in which chronically elevated TH induced profound thyrotoxicosis that resulted in early termination of hyperthyroid Cre-positive mice (Figure 6). The Cre-negative control mice receiving this $\mathrm{TH}$ dose for the same duration showed reduced rotarod performance starting immediately after treatment, consistent with Cre-positive animals, but did not show any further declines in performance (Supplemental Figure 8A); the same TH dosage has been previously dosed in healthy animals for 12 weeks with no obvious detrimental effects (24). However, on the background of demyelination in the iCKOMyrf model, chronic TH administration had additional and more severe adverse effects. Our data demonstrated that chronic TH administration inhibited OPC proliferation, leading to reduced oligodendrocyte and myelin production (Figures 6 and 7). This suggests that, in addition to the well-described TH activity promoting OPC to oligodendrocyte transition, TH can also negatively regulate OPC viability and/or proliferation. This is consistent with a recent finding that transient hypothyroidism prior to the differentiation phase had a remyelination benefit through increased production of OPCs (49).

Use of iCKO-Myrf mice as a model of myelin repair provides an improved platform for identifying therapeutics. A major challenge for identifying molecules that will be effective myelin repair agents in the clinic is the quality of available rodent models for studying remyelination. All FDA-approved strategies for MS are immunomodulatory, and the EAE model, which recapitulates the immune component of the disease, has been widely used for the evaluation of immune system-targeting therapies. Many EAE models are associated with primary axonal damage, limiting their utility for studying myelin repair, which requires intact axons (50). Remyelination is typically studied in gliotoxin models, such as the lysolecithin and cuprizone models employed in this study (Figure 2). These models have the advantage of generating primary demyelination in a relatively fast and reproducible manner. However, unlike the EAE model, there are no reproducible clinical signs associated with localized demyelination and the endogenous repair processes are rapid. 
In the cuprizone model, remyelination is coincident with ongoing demyelination, which can make identifying therapeutic benefit more challenging (34). A genetic model of demyelination based on diphtheria toxin-induced oligodendrocyte cell death showed widespread CNS demyelination followed by near-complete recovery with 10 weeks (51), but this model has not been used to evaluate myelin repair agents.

In this study, we have developed and characterized the iCKO-Myrf strain as a valuable model for studying primary demyelination and subsequent remyelination. This model has the following advantages over existing immune-based and gliotoxin models. (a) Demyelination is a single phase of the model triggered by genetic ablation of Myrf only in mature oligodendrocytes, preserving nascent and existing OPCs for remyelination. (b) Distinct demyelination and remyelination phases correlate with clinical signs that can be observed through a simple clinical scoring system or rotarod testing. (c) The incomplete recovery $(\sim 50 \%)$ provides a large window for observing pro-remyelinating therapeutic effects, as observed here with sobetirome and Sob-AM2.

The iCKO-Myrf model presented here involves a 24-week in-life phase with 22 weeks of remyelination therapy treatment. This is in contrast to traditional demyelinating models, which typically have shorter in-life phases and treatment durations of less than 3 weeks. One advantage of the longer duration treatment period is that it better reflects chronic therapy for a progressive disease such as MS, and the 22-week treatment period gives information on the tolerance of both drug and dose in a chronic treatment setting, as this dosing duration is similar to that of chronic toxicology studies in rodents. In our study, mice (both Cre negative and Cre positive) treated with sobetirome and Sob-AM2 for 22 weeks at a dose that provided therapeutic benefit showed no observed drug related adverse clinical signs (Figures 4 and 8 and Supplemental Figure 8B).

In addition, we have demonstrated that the iCKO-Myrf model is compatible with in vivo MRI imaging. MTR measurements are not specific for myelin content but have been previously demonstrated to correlate well with myelin content in the cuprizone model (34) and similarly track with histology in the iCKO-Myrf model (Figure 8). In vivo whole-brain imaging offers the opportunity to view images in real time and to serially track changes throughout the brain in the same cohort of mice through the entire disease time course. Thus, we are able to demonstrate that both control and thyromimetic-treated mice are equally affected by demyelination at week 10, supporting the conclusion that the differences observed at week 24 are due to thyromimetic-induced faster myelin repair (Figure 8 and Supplemental Figure 6).

Conclusion. The role of TH in driving OPC maturation during development is well established, but the potential of this pathway for promoting remyelination in a clinical setting has been underexplored due to the adverse effects associated with excessive TH exposure. We have shown that the selective T3 agonist sobetirome, which is unencumbered by the adverse effects of excess $\mathrm{TH}$, stimulates myelin repair in 3 independent mouse demyelinating models. Further, both sobetirome and Sob-AM2, a CNS-selective prodrug of sobetirome, show therapeutic benefit in the iCKO-Myrf genetic model. We have also shown that the iCKO-Myrf mouse represents a compelling model for the evaluation of myelin repair agents in which CNS demyelination and remyelination can be correlated to clinical decline and recovery. Sobetirome, Sob-AM2 (38), and other recently developed sobetirome derivatives $(20,21,52)$ are the only thyromimetics reported to date that distribute to the CNS from systemic administration, making this subset of selective TH agonists suitable for clinical evaluation in demyelinating diseases such as MS.

\section{Methods}

Reagents. Tamoxifen (MilliporeSigma) was dissolved in corn oil (MilliporeSigma) at $20 \mathrm{mg} / \mathrm{ml}$ with incubation for 1 hour at $37^{\circ} \mathrm{C}$, and was prepared fresh each week. Sobetirome and T3 were prepared at concentrations suitable for an i.p. injection of $150 \mu \mathrm{l}$ per $30 \mathrm{~g}$ mouse. Sobetirome was synthesized in the Scanlan Laboratory as previously described $(53,54)$. T3 (MilliporeSigma) drug stocks were prepared at $2.0 \mathrm{or} 5.0 \mathrm{mg} /$ $\mathrm{ml}$ in $40 \mathrm{mM} \mathrm{NaOH}$ in saline and diluted into saline to obtain final solutions of $0.4 \mathrm{mg} / \mathrm{ml}$ or $1.0 \mathrm{mg} / \mathrm{ml} \mathrm{T} 3$ (corresponding to 0.4 or $1.0 \mathrm{mg} / \mathrm{kg}$ body weight). Sobetirome drug stocks were prepared by dissolving sobetirome in DMSO at $2 \mathrm{mg} / \mathrm{ml}$ or $10 \mathrm{mg} / \mathrm{ml}$ and then diluting in saline to obtain a final concentration of 0.8 or $4 \mathrm{mg} / \mathrm{ml}$ solution in $40 \%$ DMSO (corresponding to 1 or $5 \mathrm{mg} / \mathrm{kg}$ body weight dose). Vehicles were prepared containing $8 \mathrm{mM} \mathrm{NaOH}$ in saline or 40\% DMSO in saline. Sobetirome was compounded into chow at a dose of $0.4 \mathrm{mg} / \mathrm{kg}$ chow and Sob-AM2 at a dose of $0.42 \mathrm{mg} / \mathrm{kg}$ chow, which corresponds to nominal daily dose of 80 and $84 \mu \mathrm{g} / \mathrm{kg}$. These doses were determined empirically as well tolerated for chronic oral exposure (24).

Animal husbandry. Mice used in the qPCR, lysolecithin, and cuprizone experiments were male C57BL6/J mice (8-10 weeks old, Jackson Laboratories). Only male mice were used in these experiments, as cycling 
estrogen levels in female mice can interact and complicate the effects observed from treatment with $\mathrm{TH}$ or thyromimetics in short-term experiments. The iCKO-Myrf (Myrfllf; Plp1-CreERT) mice were generated by crossing the Myrfl/ft line (33) to the PLP-CreERT line (55). The resulting line (32) was bred at Oregon Health $\&$ Science University (OHSU). For these long-term experiments, both male and female mice were used. The mice were housed in 12-hour/12-hour dark/light cycles with chow provided ad libitum.

OPC culture and assays. OPCs were isolated from P7 Sprague Dawley rats by immunopanning and cultured as previously described (26). Briefly, the dissociated cortices were passed over petri dishes coated with antibodies from Ran-2 and GalC hybridomas to deplete astrocytes and postmitotic oligodendrocytes, respectively, and were then placed on a dish coated with $\mathrm{O} 4$ hybridoma supernatant. The adherent cells (comprising $\sim 95 \% \mathrm{O}^{+}, \mathrm{GalC}^{-} \mathrm{OPCs}$ ) were released by trypsinization and collected. OPCs were expanded for 1 week in modified SATO medium $(26,56)$.

For differentiation assays, the expanded OPCs were replated on poly-d-lysine coated coverslips in 24-well plates at 10,000 cells per well in the above media supplemented with $50 \mathrm{nM}$ T3 (MilliporeSigma), $50 \mathrm{nM}$ sobetirome, or vehicle $(0.1 \%$ DMSO) for 96 hours. Cells were fixed with $4 \%$ paraformaldehyde (PFA) and stained overnight with rat anti-MBP (Millipore, MAB386, 1:500) and rabbit anti-NG2 (Millipore, AB5320, 1:500) in 0.1\% Triton X-100 and 10\% normal goat serum in PBS. Coverslips were washed and stained with Alexa Fluor 488-conjugated anti-rabbit and 555-conjugated anti-rat (Thermo Fisher, A27034 and A21434). Images of 4 random fields of view were taken per coverslip using a Zeiss Axio Imager M2 with a $\times 20$ objective. Images were scored for the number of NG2-positive OPCs and MBP-positive oligodendrocytes by an investigator blinded to experimental condition.

For the qPCR experiments, the OPCs were seeded in a 6-well plate at $1.6 \times 10^{6}$ cells/well in the absence of PDGF and $2.4 \times 10^{5}$ cells/well in the presence of PDGF. Under both conditions, the OPCs were incubated with vehicle (DMSO), $50 \mathrm{nM} \mathrm{T3}$, or $50 \mathrm{nM}$ sobetirome. The cells were incubated under these conditions for 24 hours and then harvested for RNA purification as described below.

qPCR analysis. C57BL6/J mice (8-10 weeks old) were treated daily with i.p. injections for 7 days. Mice were randomly assigned to receive control vehicle injection (either $20 \% \mathrm{DMSO}$ in saline or $8 \mathrm{mM} \mathrm{NaOH}$ in saline), T3 $(1.0 \mathrm{mg} / \mathrm{kg})$, or sobetirome $(1.0 \mathrm{mg} / \mathrm{kg})$. Mice were euthanized $4-6$ hours after the final injection, and tissues were immediately submerged in 10-fold excess RNALater (Thermo Fisher) for RNA preservation. Hippocampus, striatum, and medial corpus callosum was isolated by microdissection following published methods (57).

RNA was purified using either the RNeasy Mini kit (Qiagen, for cells and microdissected brain tissue) or the PureLink RNA Mini kit with TRIzol extraction (Life Technologies, for whole brain). RNA was quantified using a Nanodrop, and cDNA was prepared with the QuantiTect Reverse Transcription kit (Qiagen). qPCR was performed on an Applied Biosciences 7500 Real-Time PCR system following the QuantiTect SYBR Green PCR kit protocols (Qiagen). Results were analyzed using the $\Delta \Delta$ Ct relative quantification (58).

Rat primers were as follows: f-Gapdh-Rn 5'-CCGCATCTTCTTGTGCAGTG-3', r-Gapdh-Rn 5'-GAGAAGGCAGCCCTGGTAAC-3'; f- $M b p-R n$ 5' TCCATCCCAAGGAAAGGGGA-3', r- $M b p-R n$ 5'-TCTGCCTCCGTAGCCAAATC-3'; f- $K l f 9-R n$ 5'-CCTCCGAAAAGAGGCACAAG-3'，r-Klf9-Rn 5'-CCGAGCGCGAGAACTTTTTA-3'; f- $H r-R n$ 5'-CAAGCCCCTAGGACTCTGGATG-3', r- $H r-R n$ 5'-CCAGACGCGCAACTGAGAG-3'.

Mouse primers were as follows: f-Gapdh-Mm 5'-CCGCATCTTCTTGTGCAGTG-3', r-Gapdh- $M m$ 5'-GAGAAGGCAGCCCTGGTAAC-3'; f-Klf9- $\mathrm{Mm}$ 5'-CCTCCGAAAAGAGGCACAAG-3'， r-Klf9Mm 5'-CCGAGCGCGAGAACTTTTTA-3'; f- $H r$ - $M m$ 5'-CACTGTGTGGCATGTGTTCC-3', r- $H r-M m$ 5'-CTCCAAGGTTCCTGCTCCAG-3'

Lysolecithin demyelination model. Intracerebral injection of lysolecithin (EMD Millipore, 440154) was performed using a stereotactic apparatus. The mice received $0.2 \mathrm{ml}$ buprenorphine prior to surgery and were anesthetized with $1.5 \%-2.5 \%$ isoflurane. The injection was located with respect to the Bregma point at the $x$ and $y$ coordinates of $+1.000 \mathrm{~mm}$ and $+1.350 \mathrm{~mm}$. The skull was thinned with drilling, and the needle was lowered $2.5 \mathrm{~mm}$, targeting the rostral corpus callosum. A microinjection pump was used to inject $2 \mu \mathrm{l}$ of $2 \%$ lysolecithin in PBS or PBS over 4 minutes. The needle was kept in place for 5 minutes before slowly withdrawing the needle at a rate of $0.2 \mathrm{~mm} / \mathrm{min}$. Mice were allowed to fully recover on a heated water pad prior to returning to home cage.

Five days after lysolecithin injection, mice were randomized and daily i.p. injections of vehicle ( $40 \%$ DMSO in saline or $8 \mathrm{mM} \mathrm{NaOH}$ in saline), T3 $(0.4 \mathrm{mg} / \mathrm{kg})$, or sobetirome $(5.0 \mathrm{mg} / \mathrm{kg})$ were initiated. 
Daily i.p. injections continued until the mice were euthanized at 10, 12, or 15 days after lysolecithin injection. Brain tissue was harvested and fixed for histology. The data in Figure 1B represent the following groups (day $5, n=11$; day $10 \mathrm{Veh}, n=7$; day $10 \mathrm{~T} 3, n=6$; day $10 \mathrm{Sob} 5, n=6$; day $12 \mathrm{Veh}, n=22$; day $12 \mathrm{~T} 3, n=6$; day $12 \mathrm{Sob} 1, n=8$; day 12 Sob $5, n=15$; day $15 \mathrm{Veh}, n=8$; day $15 \mathrm{~T} 3, n=4$; day 15 Sob5, $n=3$ ).

Cuprizone demyelination model. 10- to 14-week-old male mice were fed Teklad 2016 chow compounded with $0.2 \%$ cuprizone (Envigo). Fresh chow was provided 3 times a week for 12 weeks. Mice were then switched to normal chow, and daily i.p. injections were initiated with vehicle (40\% DMSO in saline or 8 $\mathrm{mM} \mathrm{NaOH}$ in saline), T3 $(1 \mathrm{mg} / \mathrm{kg})$, or sobetirome $(1 \mathrm{mg} / \mathrm{kg})$. After 21 days, the mice were euthanized by perfusion and brains fixed and harvested for histology.

iCKO-Myrf demyelination model. At 8 weeks of age, iCKO-Myrf mice received 5 daily i.p. injections of $100 \mu 1$ tamoxifen $(20 \mathrm{mg} / \mathrm{ml})$. Starting 2 weeks after the final tamoxifen injection, mice were administered treatment through ad lib chow and water. Mice were randomly enrolled into groups receiving control chow (Teklad 2016 diet, Envigo), chow compounded with sobetirome $(0.4 \mathrm{mg} / \mathrm{kg}$ chow, $80 \mu \mathrm{g} / \mathrm{kg}$ ), chow compounded with Sob-AM2 $(0.4 \mathrm{mg} / \mathrm{kg}$ chow, $84 \mu \mathrm{g} / \mathrm{kg})$, chow compounded with a T3/T4 mixture (2 and 6 $\mathrm{mg} / \mathrm{kg}$ chow, 0.4 and $1.2 \mathrm{mg} / \mathrm{kg})$, or control chow with hypothyroid water $(0.1 \%(\mathrm{w} / \mathrm{v})$ methimazole and $0.2 \%(\mathrm{w} / \mathrm{v})$ potassium perchlorate) $(24,37,59)$. Hypothyroidism was confirmed with the ${ }^{125} \mathrm{I}$ T4 radioimmunoassay kit (IVD Technologies). Mice were weighed every week and assigned a clinical score adapted from previous studies (32) (1: limp tail or gait tremor; 2: partial hind limb weakness; 3 : hind limb paralysis (could not hang from hind limbs); and 4: ataxia and severe hindlimb paralysis). Mice were euthanized by perfusion 10,24 , or 32 weeks after tamoxifen injection.

Rotarod analysis. Mice were trained on the rotarod 2-4 days before the first experiment with three 2-minute trials at $8 \mathrm{rpm}$. Rotarod testing was initiated at week 0 prior to tamoxifen and continued weekly through the course of the experiment. Most mice were tested with a stepwise 5-minute evaluation with 0:00-1:00 at $8 \mathrm{rpm}, 1: 00-2: 00$ at $16 \mathrm{rpm}, 2: 00-3: 00$ at $24 \mathrm{rpm}, 3: 00-4: 00$ at $32 \mathrm{rpm}$ and 4:00-5:00 at $40 \mathrm{rpm}$ (Gargrish). Each ramp step (8-16 rpm, etc.) was performed over 3 seconds. The hypothyroid and comparable control groups were tested with a modified program on a different rotarod apparatus (IITC Life Sciences), in which the speed was steadily increased during 0:00-4:00 from 8-40 rpm and then held at $40 \mathrm{rpm}$ from 4:00-5:00. Latency (time to fall) was recorded for 3 trials each week, and mice were allowed to rest in their cages for at least 15 minutes between each trial.

Summary analysis of the data was performed in 2 ways. A curve was fit to the data from each individual mouse, and the time point (in weeks) at which the mouse showed sustained recovery was calculated. Sustained recovery was defined as consistent rotarod performances above 60 seconds latency for the remainder of the experiment. If the mouse did not recover above 60 seconds, it was assigned 24 weeks. The average recovery fraction was determined by averaging the rotarod latencies from weeks 18-24 by the initial latencies in weeks $0-4$ before decline.

Histology and immunofluorescence. For the lysolecithin experiments, brains were removed immediately after euthanasia, immersed in 4\% PFA, and microwaved for 1 hour using a Biowave Pro+ (Ted Pella). For the cuprizone and iCKO-Myrf experiments, mice were anesthetized with isoflurane and perfused transcardially with $10 \mathrm{ml}$ PBS followed by $40-50 \mathrm{ml}$ of 4\% PFA in PBS. Brains were removed and post-fixed for 24 hours in 4\% PFA prior to storage in PBS. Free-floating sections (40 or 50 microns) of brain were obtained using a Vibratome. For BlackGold staining (EMD Millipore, AG400), sections were mounted on positively charged slides and dried overnight at room temperature prior to staining following the manufacturer's protocol with the following modifications. For the lysolecithin experiment, sections were stained for 16-20 minutes at $60^{\circ} \mathrm{C}$. For the cuprizone and iCKO-Myrf experiments, sections were stained for 4-5 hours at room temperature. Cresyl violet was used occasionally as a counterstain; however, for lesion volume or intensity quantifications, no counterstain was used.

For immunofluorescence, all incubation steps were performed with gentle agitation on a rocker at room temperature unless otherwise noted. Antibodies were specific for PDGFR $\alpha$ (R\&D Systems, AF1062, 1:100), ASPA (EMD Millipore, ABN1698, 1:500), and MBP (EMD Millipore, MAB386, 1:500). Sections were permeabilized by incubation with $0.05 \%$ Triton X-100 in PBS for 30 minutes, followed by three 5-minute washes in PBS. Then sections were incubated in blocking buffer (3\% BSA [MilliporeSigma], $0.5 \%$ fish skin gelatin [BioWorld] in PBS) for 2 hours and transferred to primary antibody solution prepared in blocking buffer for 1 hour followed by overnight incubation at $4^{\circ} \mathrm{C}$. 
The next day, following three 5-minute washes with PBS, the sections were transferred to a secondary antibody solution (Life Technologies, A21208, A21447, and A31572, 1:200) containing DAPI (0.5 $\mu \mathrm{g} /$ $\mathrm{ml}$ final) in PBS for 1 hour followed by overnight incubation at $4^{\circ} \mathrm{C}$. The third day, the sections were washed in PBS 3 times for 5 minutes, mounted with ProGold antifade mounting solution (Life Technologies), and allowed to dry flat for 2-3 days prior to imaging.

EdU labeling. The iCKO-Myrf mice were induced with tamoxifen as described above and started on treatment chow or water 2 weeks later. Starting at 4 weeks after tamoxifen, mice were administered EdU dissolved in drinking water at a final concentration of $0.2 \mathrm{mg} / \mathrm{ml}$, and the water was replaced twice per week. Mice were euthanized 6 weeks later (week 10 after tamoxifen) and tissues were processed as described above for immunofluorescence imaging of PDGFR $\alpha$ and ASPA. Prior to mounting the sections, EdU was labeled with the Alexa Fluor 488 Click-iT EdU Imaging Kit (Invitrogen) following the provided protocol.

Microscopy and analysis. Lysolecithin lesions were imaged with the Zeiss ApoTome 2 at $\times 10$ magnification; the images were $1394 \times 1044$ microns $(1292 \times 968$ pixels $)$, and multiple images were stitched together if necessary to obtain the whole lesion. Brain sections stained with BlackGold for the iCKO-Myrf experiment were imaged at $\times 20$ (0.5 microns per pixel) with a whole-slide imager (Leica Aperio AT2). Two sections were analyzed for each mouse per region. For analysis, color images were converted to 8-bit images, and threshold values were set empirically for each group of samples based on an age-matched Cre-negative mouse. Two thresholds were set for each set of samples; the first included only intense staining in the white matter tracts, and the second included all white and gray matter staining (Supplemental Figure 7A). All comparisons shown are between samples stained in the same batch. For the sobetirome, hypothyroid, and relevant control sections, the positive (myelin) threshold was set from 0 to 70 (rostral) or from 0 to 100 (caudal) for the white matter tracts and from 0 to 160 (rostral) or from 0 to 120 (caudal) for gray matter myelin. For Sob-AM2 and related control images, the threshold was set from 0 to 75 for white matter tracts and 0 to 180 (or 0 to 200 ) for gray matter myelin.

Brains stained for immunofluorescence were imaged with a Zeiss Laser-Scanning Confocal Microscopy 780 . The cortex and hippocampus were imaged with a $\times 10 /$ NA 0.45 objective $(425 \times 425$ microns, $1024 \times 1024$ pixels $)$, and the medial caudal corpus callous was imaged a $\times 20 /$ NA 0.8 objective $(850 \times 850$ microns, $1024 \times 1024$ pixels). For all images, $Z$-stacks (every 4 microns) were acquired, and 2 sections were analyzed for each mouse. For analysis, the $Z$-stacks were compressed into a single maximum intensity projection image. PDGFR $\alpha$-, ASPA-, or EdU-labeled cells within the region of interest were manually counted. For MBP, the images were thresholded and the percentage of positive MBP staining within the region of interest was quantified. For the hippocampus, the positive (myelin) threshold was set from 12,000 to 65,535 and for the cortex, it was set from 20,000 to 65,535

$E M$. Mice were anesthetized with isoflurane and perfused transcardially with $10 \mathrm{ml}$ PBS followed by $40-50 \mathrm{ml}$ of $4 \%$ PFA and $2.5 \%$ glutaraldehyde in $0.1 \mathrm{M}$ sodium cacodylate, $\mathrm{pH} 7.4$. The brain or spinal column was removed and post-fixed in the same solution for 1 week prior to removal of the spinal cord. For the cuprizone experiments, a $1 \times 1 \times 5 \mathrm{~mm}$ block of caudal corpus callosum was dissected from the brain. For the iCKO-Myrf experiment, the lumbar spinal cord was isolated. Immediately before embedding, the tissue was post-fixed in buffer containing $0.05 \mathrm{M}$ sodium cacodylate buffer ( $\mathrm{pH} 7.4$ ), $0.05 \mathrm{M}$ sucrose, $0.25 \%$ (w/v) calcium chloride, $1.5 \%$ PFA, and 1.5\% glutaraldehyde. The tissue was embedded in plastic using Biowave Pro+ (Ted Pella). All embedding steps were performed in the microwave and included post-fixing in $2 \%$ osmium $-1.5 \%$ potassium ferrocyanide (EMS), rinsing in distilled water, staining with $0.5 \%$ uranyl acetate (EMS), dehydrating with series of acetone (20\%,35\%, 50\%, 75\%, 95\%, and $100 \%)$, infiltrating in a mixture of acetone/resin and finally embedding in resin (Spurr and Eponate 12).

Ultrathin sections $(70 \mathrm{~nm})$ were stained with 5\% uranyl acetate (10 minutes at room temperature, EMS), washed and stained with Reynold's lead citrate (5 minutes at room temperature, EMS), and dried on Nickel grids. The sections were examined using a FEI Tecnai T12 transmission electron microscope with a 16 Mpixel camera using AMT software (Advanced Microscopy Techniques Corp.). A cross section of the caudal corpus callosum was imaged at the midline. Images were obtained at $\times 4800,3$ representative images were chosen, and the number of myelinated axons was quantified. For the spinal cords, 8 images at $\times 2900$ were acquired per section from predetermined ventrolateral location on both sides of midline. The number of total axons and myelinated axons were counted in two randomly chosen images.

G-ratios were measured using the integrated morphometry analysis tool in MetaMorph software or using Fiji software. The g-ratios were calculated from the circumferences of axon and myelinated axon. 
Effective axon diameter was calculated by dividing the axon circumference by pi, and the calculated axon diameters were plotted versus g-ratios. Approximately 100-200 axons for each treatment were included in the analysis. Demyelinated axons were not included in the analysis as their g-ratios equal 1.

MRI. In vivo MRI was performed longitudinally on 6 Cre-positive mice (3 treated with SobAM2) at 10, 15 , and 24 weeks after tamoxifen using an $11.75 \mathrm{~T}$ horizontal bore instrument (Bruker Biospin) equipped with a high-performance gradient coil (9-cm inner diameter) and radiofrequency volume coil transmitter and custom-built surface receiver. Mice were initially sedated at 5\% isoflurane and maintained throughout the experiment at $1 \%-2 \%$, adjusted to maintain respiration, in $100 \%$ oxygen. Rectal temperature, respiration rate, end-tidal pulse oximetry, and heart rate were monitored throughout imaging using a small-animal physiological monitor (SA Instruments Inc.). Forced warm air was circulated through the magnet bore to maintain body temperature of $37^{\circ} \mathrm{C} \pm 1^{\circ} \mathrm{C}$. A custom-built nose-cone delivered isoflurane and secured the sedated animal to reduce motion artifacts.

Magnetization transfer (MT) MRI data were acquired using whole-brain 3D gradient-recalled echo (GRE) sequences, with $2.5 \mathrm{~ms}$ TE, $30 \mathrm{~ms}$ TR, $10^{\circ} \mathrm{FA}$; pulsed MT saturation pulse of Gauss shape, 20-ms duration, 0.01-ms interpulse delay, 137- $\mathrm{Hz}$ bandwidth, $\mathrm{B}_{1}$ field strength: $7.8 \mu \mathrm{T}$ (corresponding to $1000^{\circ}$ effective FA), and offset frequency $\Delta:+4 \mathrm{kHz}$ for MT saturation, and $\Delta:+100 \mathrm{kHz}$ for no MT saturation. All 3D GRE MT sequences were acquired at $100-\mu \mathrm{m}$ isotropic resolution and full-brain coverage (field of view $1.92 \mathrm{~cm} \times 1.44 \mathrm{~cm} \times 0.96 \mathrm{~cm}$ ). MT image processing and MTR map calculation were performed as previously described (34).

Statistics. The sample sizes were determined based on other similar published studies and our preliminary data. Mice were randomly assigned to control or treatment groups. Statistical differences between vehicle/control and treatment groups were compared using 2-tailed, unpaired $t$ tests or 1-way ANOVA with Dunnett's post-test, as indicated in the figure legends. Exact $P$ values and $n$ for each group are also indicated in the figures, figure legends, or Methods. $P$ values of less than or equal to 0.05 are considered significant. For the cuprizone and lysolecithin experiments, the different vehicles $(8 \mathrm{mM} \mathrm{NaOH}$ in saline or $40 \%$ DMSO in saline) showed no differential effects, and the data from the different vehicles were combined into a single control group. Investigators were blinded during clinical scoring and image analysis.

Study approval. All experiments were approved by IACUC committees at the VA Portland Health Care System (lysolecithin experiments) or OHSU (all other experiments).

\section{Author contributions}

$\mathrm{MDH}, \mathrm{TSS}, \mathrm{DB}$, and BE conceived of the project and experiments, and IJT, PC, GM, TB, SJF, and JMM provided advice on experimental design. BE performed the OPC cultures. IJT performed the MRI experimental design, acquisition, and analysis. $\mathrm{MDH}$ and LLK performed $\mathrm{qPCR}$ experiments and analysis. MDH, TB, LLK, MDS, MJD, SVL, HM, DG, EP, and TB conducted the in vivo experiments. MDH, TB, LLK, PC, EC, DG, MDS, and HM prepared and analyzed tissue samples. TB, SJF, and JMM synthesized sobetirome and Sob-AM2. MDH and TSS wrote the manuscript with input from IJT, DB, and BE.

\section{Acknowledgments}

The research was supported by the NIH (DK52798 to TSS), the National Multiple Sclerosis Society (NMSS; RG 5199A4 and RG-1607-25053 to DB; RG 5106A1/1 to BE), the Race to Erase MS (to DB), the OHSU Laura Fund for Innovation in Multiple Sclerosis (to DB and TSS), and gifts from the Ogg and Hoffman families (to TSS, BE, and DB). MDH received postdoctoral funding from the NIH (2T32DK007680-21) and the NMSS (FG 2023A 1/2) with partial support from the Dave Tomlinson Research Fund. IJT received postdoctoral support from the NMSS (FG-16-7-25259). We would like to thank the following core/shared resource facilities at OHSU: Advanced Light Microscopy Core and Electron Microscopy Core (both supported by the NIH, P30 NS061800), the Knight BioLibrary, Mike Lasarev in the Biostatistics \& Design Program for data analysis expertise (partially supported by the NIH, UL1TR002369), and the Advanced Imaging Research Center (partially supported by the NIH [S10 RR027694], the W.M. Keck Foundation, and Oregon Opportunity Fund). This material is the result of work supported with resources and the use of facilities at the VA Portland Health Care System, and the contents of this study do not represent the views of the Department of Veteran Affairs or the United States government. 
Address correspondence to: Thomas S. Scanlan, Department of Physiology and Pharmacology, L334; 3181 SW Sam Jackson Park Road, Oregon Health \& Science University, Portland, Oregon 97239, USA. Phone: 503.494.9292; Email: scanlant@ohsu.edu.

1. Wolswijk G. Chronic stage multiple sclerosis lesions contain a relatively quiescent population of oligodendrocyte precursor cells. J Neurosci. 1998;18(2):601-609.

2. Chang A, Nishiyama A, Peterson J, Prineas J, Trapp BD. NG2-positive oligodendrocyte progenitor cells in adult human brain and multiple sclerosis lesions. J Neurosci. 2000;20(17):6404-6412.

3. Billon N, Jolicoeur C, Tokumoto Y, Vennström B, Raff M. Normal timing of oligodendrocyte development depends on thyroid hormone receptor alpha 1 (TRalpha1). EMBO J. 2002;21(23):6452-6460.

4. Barres BA, Lazar MA, Raff MC. A novel role for thyroid hormone, glucocorticoids and retinoic acid in timing oligodendrocyte development. Development. 1994;120(5):1097-1108.

5. Schoonover $\mathrm{CM}$, et al. Thyroid hormone regulates oligodendrocyte accumulation in developing rat brain white matter tracts. Endocrinology. 2004;145(11):5013-5020.

6. Baas D, Bourbeau D, Sarliève LL, Ittel ME, Dussault JH, Puymirat J. Oligodendrocyte maturation and progenitor cell proliferation are independently regulated by thyroid hormone. Glia. 1997;19(4):324-332.

7. Gao FB, Apperly J, Raff M. Cell-intrinsic timers and thyroid hormone regulate the probability of cell-cycle withdrawal and differentiation of oligodendrocyte precursor cells. Dev Biol. 1998;197(1):54-66.

8. Najm FJ, et al. Drug-based modulation of endogenous stem cells promotes functional remyelination in vivo. Nature. 2015;522(7555):216-220.

9. Mei F, et al. Micropillar arrays as a high-throughput screening platform for therapeutics in multiple sclerosis. Nat Med. 2014;20(8):954-960

10. Deshmukh VA, et al. A regenerative approach to the treatment of multiple sclerosis. Nature. 2013;502(7471):327-332.

11. Silvestroff L, Bartucci S, Pasquini J, Franco P. Cuprizone-induced demyelination in the rat cerebral cortex and thyroid hormone effects on cortical remyelination. Exp Neurol. 2012;235(1):357-367.

12. Franco PG, Silvestroff L, Soto EF, Pasquini JM. Thyroid hormones promote differentiation of oligodendrocyte progenitor cells and improve remyelination after cuprizone-induced demyelination. Exp Neurol. 2008;212(2):458-467.

13. D'Intino G, et al. Triiodothyronine administration ameliorates the demyelination/remyelination ratio in a non-human primate model of multiple sclerosis by correcting tissue hypothyroidism. J Neuroendocrinol. 2011;23(9):778-790.

14. Fernandez M, et al. Thyroid hormone administration enhances remyelination in chronic demyelinating inflammatory disease. Proc Natl Acad Sci USA. 2004;101(46):16363-16368.

15. Calza L, Fernandez M, Giuliani A, Aloe L, Giardino L. Thyroid hormone activates oligodendrocyte precursors and increases a myelin-forming protein and NGF content in the spinal cord during experimental allergic encephalomyelitis. Proc Natl Acad Sci USA. 2002;99(5):3258-3263.

16. Harsan LA, et al. Recovery from chronic demyelination by thyroid hormone therapy: myelinogenesis induction and assessment by diffusion tensor magnetic resonance imaging. J Neurosci. 2008;28(52):14189-14201.

17. Zhang M, Zhan XL, Ma ZY, Chen XS, Cai QY, Yao ZX. Thyroid hormone alleviates demyelination induced by cuprizone through its role in remyelination during the remission period. Exp Biol Med (Maywood). 2015;240(9):1183-1196.

18. Calzà L, Baldassarro VA, Fernandez M, Giuliani A, Lorenzini L, Giardino L. Thyroid Hormone and the White Matter of the Central Nervous System: From Development to Repair. Vitam Horm. 2018;106:253-281.

19. Scanlan TS. Sobetirome: a case history of bench-to-clinic drug discovery and development. Heart Fail Rev. 2010;15(2):177-182.

20. Ferrara SJ, et al. Ester-to-amide rearrangement of ethanolamine-derived prodrugs of sobetirome with increased blood-brain barrier penetration. Bioorg Med Chem. 2017;25(10):2743-2753.

21. Placzek AT, Ferrara SJ, Hartley MD, Sanford-Crane HS, Meinig JM, Scanlan TS. Sobetirome prodrug esters with enhanced blood-brain barrier permeability. Bioorg Med Chem. 2016;24(22):5842-5854.

22. Manzano J, Morte B, Scanlan TS, Bernal J. Differential effects of triiodothyronine and the thyroid hormone receptor beta-specific agonist GC-1 on thyroid hormone target genes in the brain. Endocrinology. 2003;144(12):5480-5487.

23. Baxi EG, et al. A selective thyroid hormone $\beta$ receptor agonist enhances human and rodent oligodendrocyte differentiation. Glia. 2014;62(9):1513-1529.

24. Hartley MD, Kirkemo LL, Banerji T, Scanlan TS. A Thyroid Hormone-Based Strategy for Correcting the Biochemical Abnormality in X-Linked Adrenoleukodystrophy. Endocrinology. 2017;158(5):1328-1338.

25. Najm FJ, et al. Transcription factor-mediated reprogramming of fibroblasts to expandable, myelinogenic oligodendrocyte progenitor cells. Nat Biotechnol. 2013;31(5):426-433.

26. Dugas JC, Emery B. Purification of oligodendrocyte precursor cells from rat cortices by immunopanning. Cold Spring Harb Protoc. 2013;2013(8):745-758.

27. Strait KA, Carlson DJ, Schwartz HL, Oppenheimer JH. Transient stimulation of myelin basic protein gene expression in differentiating cultured oligodendrocytes: a model for 3,5,3'-triiodothyronine-induced brain development. Endocrinology. 1997;138(2):635-641.

28. Dugas JC, Ibrahim A, Barres BA. The T3-induced gene KLF9 regulates oligodendrocyte differentiation and myelin regeneration. Mol Cell Neurosci. 2012;50(1):45-57.

29. Thompson CC, Bottcher MC. The product of a thyroid hormone-responsive gene interacts with thyroid hormone receptors. Proc Natl Acad Sci USA. 1997;94(16):8527-8532.

30. Back SA, et al. Hyaluronan accumulates in demyelinated lesions and inhibits oligodendrocyte progenitor maturation. Nat Med. 2005;11(9):966-972.

31. Bai CB, et al. A mouse model for testing remyelinating therapies. Exp Neurol. 2016;283(Pt A):330-340. 
32. Koenning M, et al. Myelin gene regulatory factor is required for maintenance of myelin and mature oligodendrocyte identity in the adult CNS. J Neurosci. 2012;32(36):12528-12542.

33. Emery B, et al. Myelin gene regulatory factor is a critical transcriptional regulator required for CNS myelination. Cell. 2009;138(1):172-185.

34. Tagge I, et al. Spatio-Temporal Patterns of Demyelination and Remyelination in the Cuprizone Mouse Model. PLoS ONE. 2016;11(4):e0152480.

35. Plemel JR, Liu WQ, Yong VW. Remyelination therapies: a new direction and challenge in multiple sclerosis. Nat Rev Drug Discov. 2017;16(9):617-634.

36. Ferrara SJ, Bourdette D, Scanlan TS. Hypothalamic-Pituitary-Thyroid Axis Perturbations in Male Mice by CNS-Penetrating Thyromimetics. Endocrinology. 2018;159(7):2733-2740.

37. Bianco AC, et al. American Thyroid Association Guide to investigating thyroid hormone economy and action in rodent and cell models. Thyroid. 2014;24(1):88-168.

38. Meinig JM, et al. Targeting Fatty-Acid Amide Hydrolase with Prodrugs for CNS-Selective Therapy. ACS Chem Neurosci. 2017;8(11):2468-2476.

39. Hartley MD, Altowaijri G, Bourdette D. Remyelination and multiple sclerosis: therapeutic approaches and challenges. Curr Neurol Neurosci Rep. 2014;14(10):485.

40. Hubler Z, et al. Accumulation of 8,9-unsaturated sterols drives oligodendrocyte formation and remyelination. Nature. 2018;560(7718):372-376.

41. Mei F, et al. Identification of the Kappa-Opioid Receptor as a Therapeutic Target for Oligodendrocyte Remyelination. J Neurosci. 2016;36(30):7925-7935.

42. Meinig JM, et al. Structure-Activity Relationships of Central Nervous System Penetration by Fatty Acid Amide Hydrolase (FAAH)-Targeted Thyromimetic Prodrugs. ACS Med Chem Lett. 2019;10(1):111-116.

43. Wei BQ, Mikkelsen TS, McKinney MK, Lander ES, Cravatt BF. A second fatty acid amide hydrolase with variable distribution among placental mammals. J Biol Chem. 2006;281(48):36569-36578.

44. Bernal J. Thyroid hormone receptors in brain development and function. Nat Clin Pract Endocrinol Metab. 2007;3(3):249-259.

45. Armour CM, Kersseboom S, Yoon G, Visser TJ. Further Insights into the Allan-Herndon-Dudley Syndrome: Clinical and Functional Characterization of a Novel MCT8 Mutation. PLoS ONE. 2015;10(10):e0139343.

46. Bárez-López S, Hartley MD, Grijota-Martínez C, Scanlan TS, Guadaño-Ferraz A. Sobetirome and its Amide Prodrug SobAM2 Exert Thyromimetic Actions in Mct8-Deficient Brain. Thyroid. 2018;28(9):1211-1220.

47. Zendedel A, et al. Regulatory effect of triiodothyronine on brain myelination and astrogliosis after cuprizone-induced demyelination in mice. Metab Brain Dis. 2016;31(2):425-433.

48. Trost SU, et al. The thyroid hormone receptor-beta-selective agonist GC-1 differentially affects plasma lipids and cardiac activity. Endocrinology. 2000;141(9):3057-3064.

49. Remaud S, et al. Transient hypothyroidism favors oligodendrocyte generation providing functional remyelination in the adult mouse brain. Elife. 2017;6:e29996.

50. Franklin RJ. Regenerative Medicines for Remyelination: From Aspiration to Reality. Cell Stem Cell. 2015;16(6):576-577.

51. Traka M, et al. A genetic mouse model of adult-onset, pervasive central nervous system demyelination with robust remyelination. Brain. 2010;133(10):3017-3029.

52. Devereaux J, Ferrara SJ, Banerji T, Placzek AT, Scanlan TS. Increasing Thyromimetic Potency through Halogen Substitution. ChemMedChem. 2016;11(21):2459-2465.

53. Chiellini G, Apriletti JW, Yoshihara HA, Baxter JD, Ribeiro RC, Scanlan TS. A high-affinity subtype-selective agonist ligand for the thyroid hormone receptor. Chem Biol. 1998;5(6):299-306.

54. Placzek AT, Scanlan TS. New synthetic routes to thyroid hormone analogs: d6-sobetirome, $3 \mathrm{H}$-sobetirome, and the antagonist NH-3. Tetrahedron. 2015;71(35):5946-5951.

55. Doerflinger NH, Macklin WB, Popko B. Inducible site-specific recombination in myelinating cells. Genesis. 2003;35(1):63-72.

56. Bottenstein JE, Sato GH. Growth of a rat neuroblastoma cell line in serum-free supplemented medium. Proc Natl Acad Sci USA. 1979;76(1):514-517

57. Spijker S. Dissection of Rodent Brain Regions. In: Li KW, ed. Neuroproteomics. New York, NY: Humana Press; $2011: 13-26$.

58. Pfaffl MW. A new mathematical model for relative quantification in real-time RT-PCR. Nucleic Acids Res. 2001;29(9):e45.

59. Hackenmueller SA, Marchini M, Saba A, Zucchi R, Scanlan TS. Biosynthesis of 3-iodothyronamine (T1AM) is dependent on the sodium-iodide symporter and thyroperoxidase but does not involve extrathyroidal metabolism of T4. Endocrinology. 2012;153(11):5659-5667. 